\title{
Normal Contact Without Adhesion
}

\subsection{Introduction}

We begin our consideration of contact phenomena with the normal contact problem. Consider two bodies pressed together by forces perpendicular to their surfaces. A prominent example is the wheel of a train on a rail. The two most important relationships that the theory of normal contact should deliver are:

1. The relationship between the normal force and the normal displacement of the body, which determines the stiffness of the contact and, therefore, the dynamic properties of the entire system.

2. The stresses occurring in the contact area, which (for example) are required for component strength analysis.

Without physical contact, there are no other contact phenomena, no friction, and no wear. Therefore, normal contact can be regarded as a basic prerequisite for all other tribological phenomena. The solution to the adhesive contact problem, the tangential contact problem, and contact between elastomers can also be reduced to the non-adhesive normal contact problem. In this sense, the non-adhesive contact problem forms a fundamental basis of contact mechanics. It should be noted that even during normal contact, a relative tangential movement between contacting surfaces can occur due to different transverse contraction of contacting bodies. As a result, friction forces between the surfaces can play a role, even for normal contact problems, and it must be specified how these tangential stresses are to be treated. The two most well-known and sudied limiting cases are, firstly, the frictionless normal contact problem and, secondly, the contact problem with complete stick. All frictionless contact problems will be referred to as "Boussinesq problems" since the famous Boussinesq solution for a cylindrical flat punch belongs to this category. The other limiting case of complete stick will be referred to as "Mossakovskii problems". 


\subsection{Boussinesq Problems (Frictionless)}

We consider the frictionless normal contact between two elastic bodies with the elasticity moduli $E_{1}$ and $E_{2}$, and Poisson's ratios $\nu_{1}$ and $\nu_{2}$, as well as shear moduli $G_{1}$ and $G_{2}$. The axisymmetric difference between the profiles will be written as $\tilde{z}=f(r)$, where $r$ is the polar radius in the contact plane. This contact problem is equivalent to the contact of a rigid indenter with the profile $\tilde{z}=f(r)$ and an elastic half-space with the effective elasticity modulus $E^{*}$ (Hertz 1882):

$$
\frac{1}{E^{*}}=\frac{1-v_{1}^{2}}{E_{1}}+\frac{1-v_{2}^{2}}{E_{2}} .
$$

The positive direction of $\tilde{z}$ is defined by the outward-surface normal of the elastic half-space. The normal component of the displacement of the medium $w$, under the influence of a concentrated normal force $F_{z}$ in the coordinate origin, is given by the fundamental solution (Boussinesq 1885):

$$
w(r)=\frac{1}{\pi E^{*}} \frac{F_{z}}{r} .
$$

Applying the superposition principle to an arbitrary pressure distribution $p(x, y)=-\sigma_{z z}(x, y)$ yields the displacement field:

$$
w(x, y)=\frac{1}{\pi E^{*}} \iint p\left(x^{\prime}, y^{\prime}\right) \frac{\mathrm{d} x^{\prime} \mathrm{d} y^{\prime}}{r}, \quad r=\sqrt{\left(x-x^{\prime}\right)^{2}+\left(y-y^{\prime}\right)^{2}} .
$$

The positive direction of the normal force and normal displacement are defined by the inward-surface normal of the elastic half-space. If we call the indentation depth of the contact $d$ and the contact radius $a$, the mixed boundary conditions for the displacement $w$ and the stresses $\sigma$ at the half-space surface (i.e., $z=0$ ) are as follows:

$$
\begin{aligned}
w(r) & =d-f(r), \quad r \leq a, \\
\sigma_{z z}(r) & =0, \quad r>a, \\
\sigma_{z r}(r) & =0 .
\end{aligned}
$$

Usually, $a$ is not known a priori, but has to be determined in the solution process. The solution of the contact problem is found by determining the pressure distribution, which satisfies (2.3) and the boundary conditions (2.4). It should be noted that the application of both the superposition principle and the boundary conditions in the form (2.4) require linearity of the material behavior as well as the half-space approximation to be met; i.e., the surface gradient must be small in the relevant area of the given contact problem in the non-deformed and deformed state. If we call the gradient $\theta$ then the condition is $\theta \ll 1$. The relative error resulting from the application of the half-space approximation is of the order of $\theta^{2}$.

For ordinarily connected contacts the non-adhesive normal contact problem was solved in its general form by Schubert (1942) (based on the paper by Föppl (1941)), 
Galin (1946), Shtaerman (1949), and Sneddon (1965). In Sect. 2.3 we will describe these solutions using the interpretation given by the method of dimensionality reduction (MDR) (Popov and Heß 2013). Naturally, it is fully equivalent to the classical solutions.

\subsection{Solution Algorithm Using MDR}

The contact of any given axially symmetric bodies can be solved very easily and elegantly with the so-called MDR. The MDR maps three-dimensional contacts to contacts with a one-dimensional array of independent springs (Winkler foundation). Despite its simplicity, all results are exact for axially symmetrical contacts. The MDR allows the study of non-adhesive and adhesive contacts, tangential contacts with friction, as well as contacts with viscoelastic media. In this section we will describe the application of the MDR for non-adhesive normal contact problems. Generalizations for other problems will be presented where appropriate in later chapters. Complete derivations can be found in works by Popov and Heß (2013, 2015), as well as in Chap. 11 in this book (Appendix).

\subsubsection{Preparatory Steps}

Solving the contact problem by way of the MDR requires two preparatory steps.

1. First, the three-dimensional elastic (or viscoelastic) bodies are replaced by a Winkler foundation. This is a linear arrangement of elements with independent degrees of freedom, with a sufficiently small distance $\Delta x$ between the elements.

In the case of elastic bodies, the foundation consists of linear-elastic springs with a normal stiffness (Fig. 2.1):

$$
\Delta k_{z}=E^{*} \Delta x,
$$

whereby $E^{*}$ is given by (2.1).

2. Next, the three-dimensional profile $\tilde{z}=f(r)$ (left in Fig. 2.2) is transformed to a plane profile $g(x)$ (right in Fig. 2.2) according to:

$$
g(x)=|x| \int_{0}^{|x|} \frac{f^{\prime}(r)}{\sqrt{x^{2}-r^{2}}} \mathrm{~d} r .
$$

Fig. 2.1 One-dimensional elastic foundation

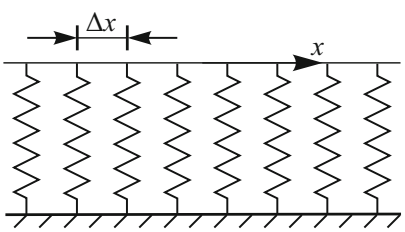


Fig. 2.2 Within the MDR the three-dimensional profile is transformed to a plane profile

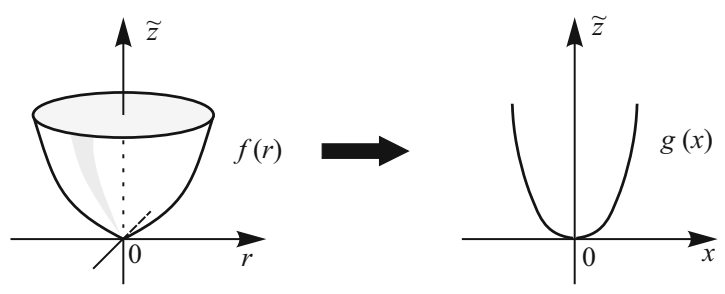

The inverse transform is:

$$
f(r)=\frac{2}{\pi} \int_{0}^{r} \frac{g(x)}{\sqrt{r^{2}-x^{2}}} \mathrm{~d} x .
$$

\subsubsection{Calculation Procedure of the MDR}

The plane profile $g(x)$ of (2.6) is now pressed into the elastic foundation with the normal force $F_{N}$ (see Fig. 2.3).

The normal surface displacement at position $x$ within the contact area is equal to the difference of the indentation depth $d$ and the profile shape $g$ :

$$
w_{1 D}(x)=d-g(x)
$$

At the boundary of the non-adhesive contact, $x= \pm a$, the surface displacement must be zero:

$$
w_{1 D}( \pm a)=0 \quad \Rightarrow \quad d=g(a) .
$$

This equation determines the relationship between the indentation depth and the contact radius $a$. Note that this relationship does not depend upon the elastic properties of the medium.

The force of a spring at position $x$ is proportional to the displacement at this position:

$$
\Delta F_{N}(x)=\Delta k_{z} w_{1 D}(x)=E^{*} w_{1 D}(x) \Delta x .
$$

Fig. 2.3 MDR substitute model for the normal contact problem

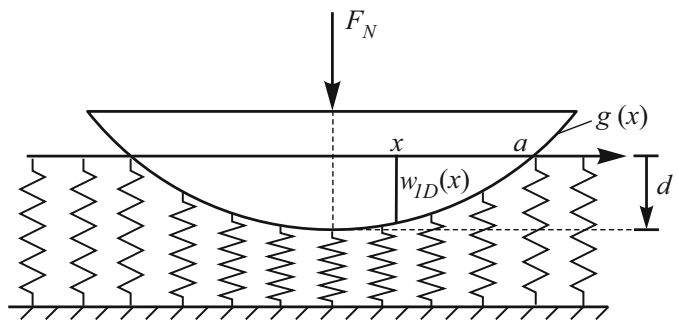


The sum of all spring forces must balance out the external normal force. In the limiting case of very small spring spacing, $\Delta x \rightarrow \mathrm{d} x$, the sum turns into an integral:

$$
F_{N}=E^{*} \int_{-a}^{a} w_{1 D}(x) \mathrm{d} x=2 E^{*} \int_{0}^{a}[d-g(x)] \mathrm{d} x .
$$

Equation (2.11) provides the normal force as a function of the contact radius and, under consideration of (2.9), of the indentation depth. Let us now define the linear force density $q_{z}(x)$ :

$$
q_{z}(x)=\frac{\Delta F_{N}(x)}{\Delta x}=E^{*} w_{1 D}(x)=E^{*}\left\{\begin{array}{ll}
d-g(x), & |x|<a \\
0, & |x|>a
\end{array} .\right.
$$

As shown in the appendix to this book, the stress distribution of the original threedimensional system can be determined from the one-dimensional linear force density via the integral transform:

$$
\sigma_{z z}(r)=-p(r)=\frac{1}{\pi} \int_{r}^{\infty} \frac{q_{z}^{\prime}(x)}{\sqrt{x^{2}-r^{2}}} \mathrm{~d} x
$$

The normal surface displacement $w(r)$ (inside as well as outside the contact area) is given by the transform:

$$
w(r)=\frac{2}{\pi} \int_{0}^{r} \frac{w_{1 D}(x)}{\sqrt{r^{2}-x^{2}}} \mathrm{~d} x .
$$

For the sake of completeness, we will provide the inverse transform to (2.13):

$$
q_{z}(x)=2 \int_{x}^{\infty} \frac{r p(r)}{\sqrt{r^{2}-x^{2}}} \mathrm{~d} r .
$$

With the MDR it is also possible to determine the displacements for a prescribed stress distribution at the surface of the half-space. First, the displacement of the Winkler foundation $w_{1 D}$ must be calculated from the stresses according to:

$$
w_{1 D}(x)=\frac{q_{z}(x)}{E^{*}}=\frac{2}{E^{*}} \int_{x}^{\infty} \frac{r p(r)}{\sqrt{r^{2}-x^{2}}} \mathrm{~d} r .
$$

Substituting this result into (2.14) allows the calculation of the three-dimensional displacements.

Equations (2.6), (2.9), (2.11), (2.13), and (2.14) completely solve the nonadhesive frictionless normal contact problem, so we state them once again in a more compact and slightly modified form: 


$$
\begin{aligned}
& g(x)=|x| \int_{0}^{|x|} \frac{f^{\prime}(r) \mathrm{d} r}{\sqrt{x^{2}-r^{2}}} \\
& d=g(a), \quad \text { if } g \text { continous at } x=a, \\
& F_{N}=2 E^{*} \int_{0}^{a}[d-g(x)] \mathrm{d} x, \\
& \sigma_{z z}(r)=-\frac{E^{*}}{\pi}\left[\int_{r}^{a} \frac{g^{\prime}(x) \mathrm{d} x}{\sqrt{x^{2}-r^{2}}}+\frac{d-g(a)}{\sqrt{a^{2}-r^{2}}}\right], \quad \text { for } r \leq a, \\
& w(r)=\frac{2}{\pi}\left\{\int_{0}^{r} \frac{[d-g(x)] \mathrm{d} x}{\sqrt{r^{2}-x^{2}}}, \quad \text { for } r<a,\right. \\
& \int_{0}^{a} \frac{[d-g(x)] \mathrm{d} x}{\sqrt{r^{2}-x^{2}}}, \text { for } r>a .
\end{aligned}
$$

In the following we will present the relationships between the normal force $F_{N}$, indentation depth $d$, and contact radius $a$, as well as the stresses and displacements outside the contact area for various technically relevant profiles $f(r)$.

\subsection{Areas of Application}

The most well-known normal contact problem is likely the Hertzian contact (see Sects. 2.5.3 and 2.5.4). While Hertz (1882) examined the contact of two parabolic bodies with different radii of curvature around the $x$-axis and $y$-axis in his work, we will consider the more specific axisymmetric case of the contact of two elastic spheres or, equivalently, of a rigid sphere and an elastic half-space. This problem occurs ubiquitously in technical applications; for example, in roller bearings, joints, or the contact between wheel and rail. Hertz also proposed using this contact for measuring material hardness in Hertzian contact, however, the stress maximum lies underneath the surface of the half-space. Therefore cones (see Sect. 2.5.2) are more suitable for this task. For punching, flat indenters (see Sect. 2.5.1) or even flat rings (see Sect. 2.5.7) are very commonly used because of the stress singularity at the edge of the contact.

These three shapes-flat, cone-shaped, and spherical indenters-essentially form the three ideal base shapes for most contacts in technical applications. Additionally, it is also of great value to examine the effects of imperfections on these base shapes, for example through manufacturing or wear. Such imperfect indenters may be truncated cones or spheres (see Sects. 2.5.9 and 2.5.10), bodies with 
rounded tips (see Sects. 2.5.11 to 2.5.13) or rounded edges (see Sect. 2.5.14), as well as ellipsoid profiles (see Sect. 2.5.5).

Furthermore, any infinitely often continuously differentiable profile can be expanded in a Taylor series. By utilizing a profile defined by a power-law (see Sect. 2.5.8), the solution for a more complex profile-assuming it satisfies the aforementioned differentiability criterion-can be constructed to arbitrary precision with the Taylor series.

Furthermore, this chapter contains profiles relevant for applications where adhesive normal contact comes into play. This includes a profile which generates a constant pressure distribution (see Sect. 2.5.5), concave bodies (see Sects. 2.5.15 and 2.5.16), or bodies with a periodic roughness (see Sect. 2.5.17). Since the next chapter dealing with adhesive normal contact reveals that the frictionless normal contact problem with adhesion can, under certain circumstances, be reduced to the non-adhesive one, we will provide the corresponding non-adhesive solutions already in this chapter, even though the practical significance of the respective problems will only become apparent later.

The contact problem is fully defined by the profile $f(r)$ and one of the global contact quantities $F_{N}, d$ or $a$. Generally, we will assume that, of these three, the contact radius is given, consequentially yielding the solution as a function of this contact radius. Should, instead of the contact radius, the normal force or the indentation depth be given, the given equations must be substituted as necessary.

\subsection{Explicit Solutions for Axially Symmetric Profiles}

\subsubsection{The Cylindrical Flat Punch}

The solution of the normal contact problem for the flat cylindrical punch of radius $a$, which can be described by the profile:

$$
f(r)= \begin{cases}0, & r \leq a, \\ \infty, & r>a\end{cases}
$$

goes back to Boussinesq (1885). The utilized notation is illustrated in Fig. 2.4. The original solution by Boussinesq is based on the methods of potential theory. The solution using the MDR is significantly simpler. The equivalent flat profile for the purposes of the MDR, $g(x)$, is given by:

$$
g(x)= \begin{cases}0, & |x| \leq a, \\ \infty, & |x|>a .\end{cases}
$$

The contact radius corresponds to the radius of the indenter. The only remaining global contact quantities to be determined are the indentation depth $d$ and the 
Fig. 2.4 Normal indentation by a cylindrical flat punch

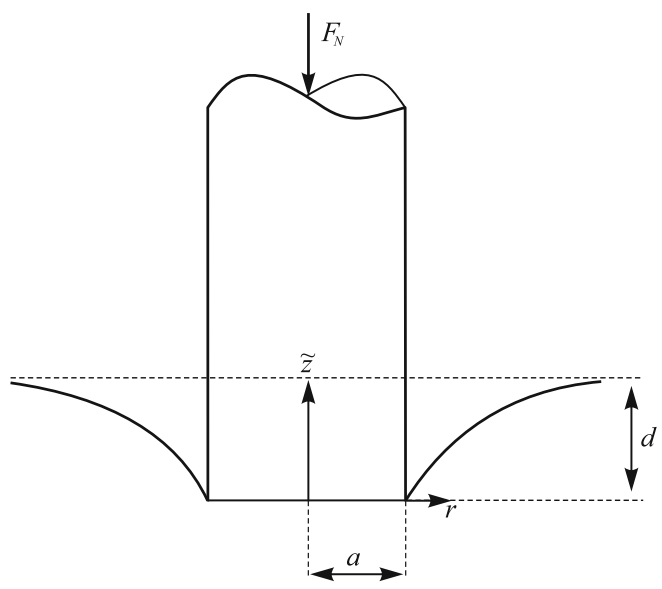

normal force $F_{N}$. For the latter we obtain:

$$
F_{N}(d)=2 E^{*} d a \text {. }
$$

Hence, the contact stiffness equals:

$$
k_{z}:=\frac{\mathrm{d} F_{N}}{\mathrm{~d} d}=2 E^{*} a .
$$

The stress distribution in the contact area and the displacements of the half-space outside the contact are, due to (2.17):

$$
\begin{aligned}
\sigma_{z z}(r ; d) & =-\frac{E^{*} d}{\pi \sqrt{a^{2}-r^{2}}}, \quad r \leq a, \\
w(r ; d) & =\frac{2 d}{\pi} \arcsin \left(\frac{a}{r}\right), \quad r>a .
\end{aligned}
$$

The average pressure in the contact is:

$$
p_{0}=\frac{F_{N}}{\pi a^{2}}=\frac{2 E^{*} d}{\pi a}
$$

The stress distribution and displacements within and outside the contact area are shown in Figs. 2.5 and 2.6.

Finally, it should be noted that, although the notation $E^{*}$ is used for the cylindrical indenter, implying a possible elasticity of both contact bodies, the aforementioned solution described previously is solely valid for rigid cylindrical indenters. While the deformation of the half-space can satisfy the conditions of the half-space approximation, this is generally not the case for the cylindrical indenter. The discrepancies which occur for elastic indenters are discussed in Sect. 2.5.19. 
Fig. 2.5 Normal pressure $p=-\sigma_{z z}$, normalized to the average pressure in the contact $p_{0}$, for the indentation by a flat cylindrical punch

Fig. 2.6 Displacement of the half-space, normalized to the indentation depth $d$, for the indentation by a flat cylindrical punch
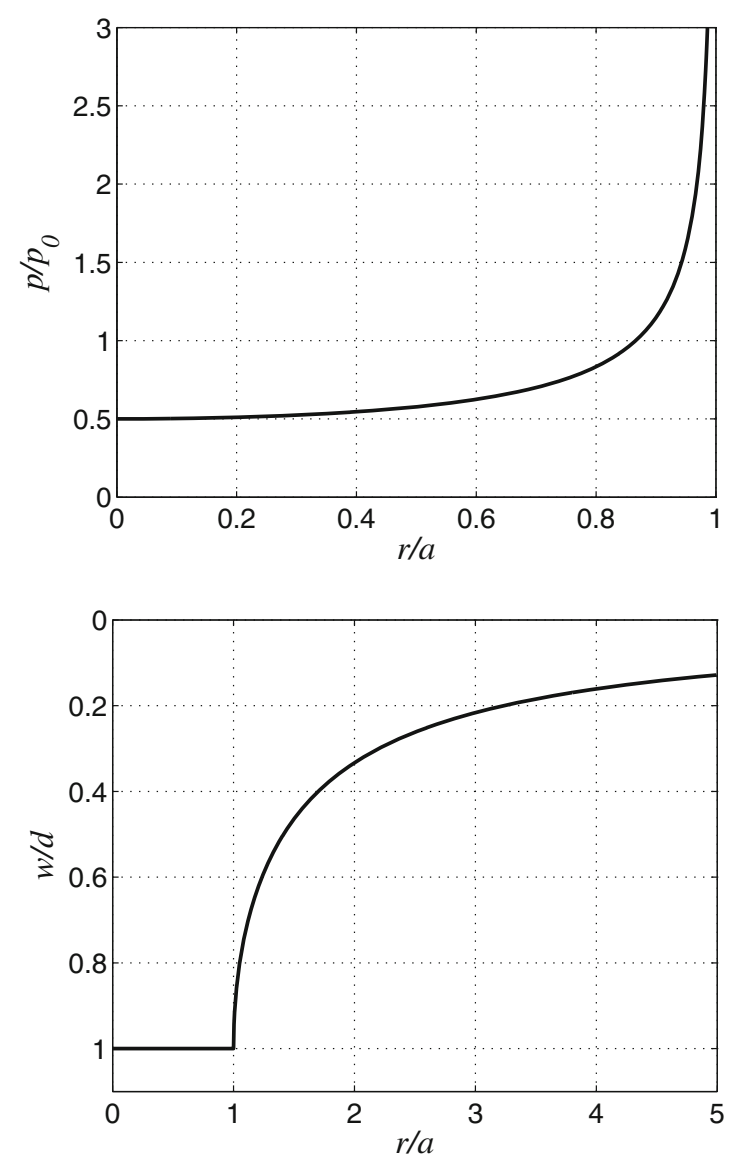

\subsubsection{The Cone}

The case of the conical indenter (see Fig. 2.7),

$$
f(r)=r \tan \theta,
$$

with a small inclination angle $\theta$, was first solved by Love (1939). He also made use of potential theory and used several tricky series expansions to obtain the solution. Again, we describe the easier way of using the MDR. The equivalent profile is given by:

$$
g(x)=|x| \tan \theta \int_{0}^{|x|} \frac{\mathrm{d} r}{\sqrt{x^{2}-r^{2}}}=\frac{\pi}{2}|x| \tan \theta .
$$

For the relationships between contact radius $a$, indentation depth $d$, normal force $F_{N}$, average pressure $p_{0}$, and for the stresses $\sigma_{z z}$ and displacements $w$, according 
Fig. 2.7 Normal indentation by a conical indenter

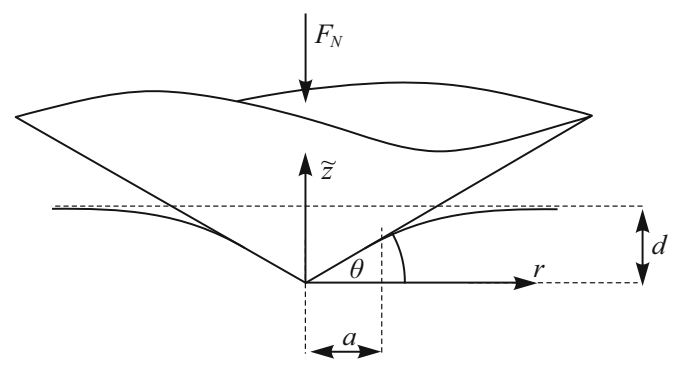

to (2.17) we obtain:

$$
\begin{aligned}
d(a) & =\frac{\pi}{2} a \tan \theta, \\
F_{N}(a) & =\pi E^{*} \tan \theta \int_{0}^{a}(a-x) \mathrm{d} x=\frac{\pi a^{2}}{2} E^{*} \tan \theta, \\
p_{0} & =\frac{1}{2} E^{*} \tan \theta, \\
\sigma_{z z}(r ; a) & =-\frac{E^{*} \tan \theta}{2} \int_{r}^{a} \frac{\mathrm{d} x}{\sqrt{x^{2}-r^{2}}}=-p_{0} \operatorname{arcosh}\left(\frac{a}{r}\right), \quad r \leq a, \\
w(r ; a) & \left.=\tan \theta \int_{0}^{a} \frac{(a-x) \mathrm{d} x}{\sqrt{r^{2}-x^{2}}}\right], \quad r>a . \\
& =a \tan \theta\left[\arcsin \left(\frac{a}{r}\right)+\frac{\sqrt{r^{2}-a^{2}}-r}{a}\right],
\end{aligned}
$$

Here $\operatorname{arcosh}(\cdot)$ denotes the area hyperbolic cosine function, which can also be represented explicitly by the natural logarithm:

$$
\operatorname{arcosh}\left(\frac{a}{r}\right)=\ln \left(\frac{a+\sqrt{a^{2}-r^{2}}}{r}\right) .
$$

The stress distribution, normalized to the average pressure in the contact, is shown in Fig. 2.8. One recognizes the logarithmic singularity at the apex of the cone. In Fig. 2.9, the displacement of the half-space normalized to the indentation depth $d$ is shown.

Finally, it should be noted-in analogy to the previous section-that although the notation $E^{*}$ is used for the conical punch as well (implying that both contacting bodies are allowed to be elastic), the previously described solution is correct without restrictions only for rigid conical indenters. While for the half-space the requirements of the half-space approximation can still be fulfilled, for the conical 
Fig. 2.8 Course of normal pressure $p=-\sigma_{z z}$, normalized to the average pressure, for indentation by a cone

Fig. 2.9 Displacement of the half-space, normalized to the indentation depth $d$, for indentation by a cone
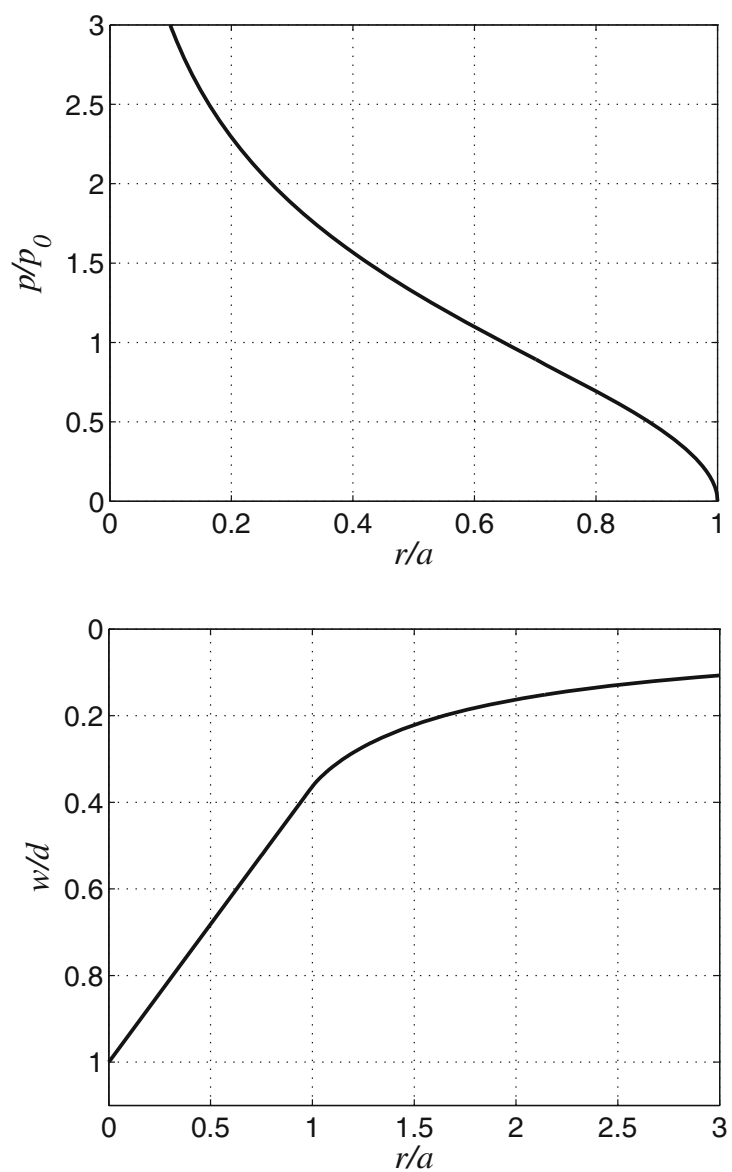

punch this is the case only for small angles $\theta$. The deviations that occur in the case of elastic indenters are addressed in Sect. 2.5.19.

\subsubsection{The Paraboloid}

The solution to the problem illustrated in Fig. 2.10 goes back to the classical work of Hertz (1882), although he studied the generalized problem of an elliptic contact area. Hertz made use of potential theory too. For a small contact radius $a$ compared to the radius of the sphere $R$, the profile shape in the contact is characterized by the parabola:

$$
f(r)=\frac{r^{2}}{2 R} .
$$


Fig. 2.10 Normal indentation by a parabolic indenter

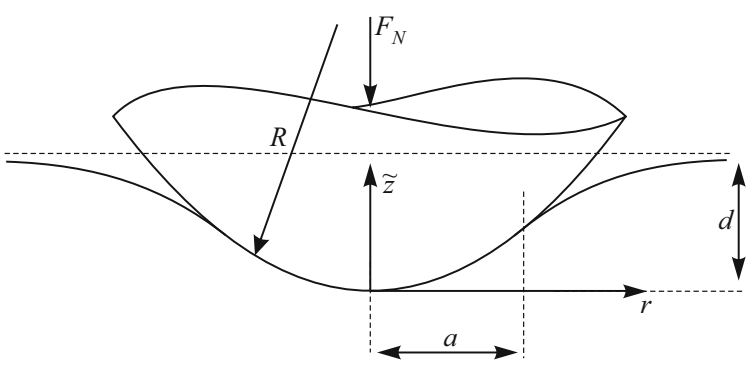

The solution of the contact problem as per (2.17) is given by:

$$
\begin{aligned}
g(x) & =\frac{|x|}{R} \int_{0}^{|x|} \frac{r \mathrm{~d} r}{\sqrt{x^{2}-r^{2}}}=\frac{x^{2}}{R}, \\
d(a) & =\frac{a^{2}}{R}, \\
F_{N}(a) & =\frac{2 E^{*}}{R} \int_{0}^{a}\left(a^{2}-x^{2}\right) \mathrm{d} x=\frac{4}{3} \frac{E^{*} a^{3}}{R} .
\end{aligned}
$$

And by:

$$
\begin{aligned}
\sigma_{z z}(r ; a) & =-\frac{2 E^{*}}{\pi R} \int_{r}^{a} \frac{x \mathrm{~d} x}{\sqrt{x^{2}-r^{2}}}=-\frac{2 E^{*}}{\pi R} \sqrt{a^{2}-r^{2}}, \quad r \leq a, \\
w(r ; a) & =\frac{2}{\pi R} \int_{0}^{a} \frac{\left(a^{2}-x^{2}\right) \mathrm{d} x}{\sqrt{r^{2}-x^{2}}} \\
& =\frac{a^{2}}{\pi R}\left[\left(2-\frac{r^{2}}{a^{2}}\right) \arcsin \left(\frac{a}{r}\right)+\frac{\sqrt{r^{2}-a^{2}}}{a}\right], \quad r>a .
\end{aligned}
$$

The average pressure in the contact is equal to:

$$
p_{0}=\frac{4 E^{*} a}{3 \pi R} .
$$

The stress curve normalized to this pressure and the displacement curve normalized to the indentation depth $d$ are shown in Figs. 2.11 and 2.12, respectively. In this normalized representation, the curves of the contact quantities are independent of the curvature radius $R$.

\section{Stresses within the Half-Space}

As Huber (1904) demonstrated, the stresses inside the half-space can also be calculated for this contact problem. After a lengthy calculation, he provided the 
Fig. 2.11 Normal pressure curve $p=-\sigma_{z z}$, normalized to the average pressure in the contact, for the indentation by a paraboloid

Fig. 2.12 Displacement of the half-space, normalized to the indentation depth $d$, for the indentation by a paraboloid
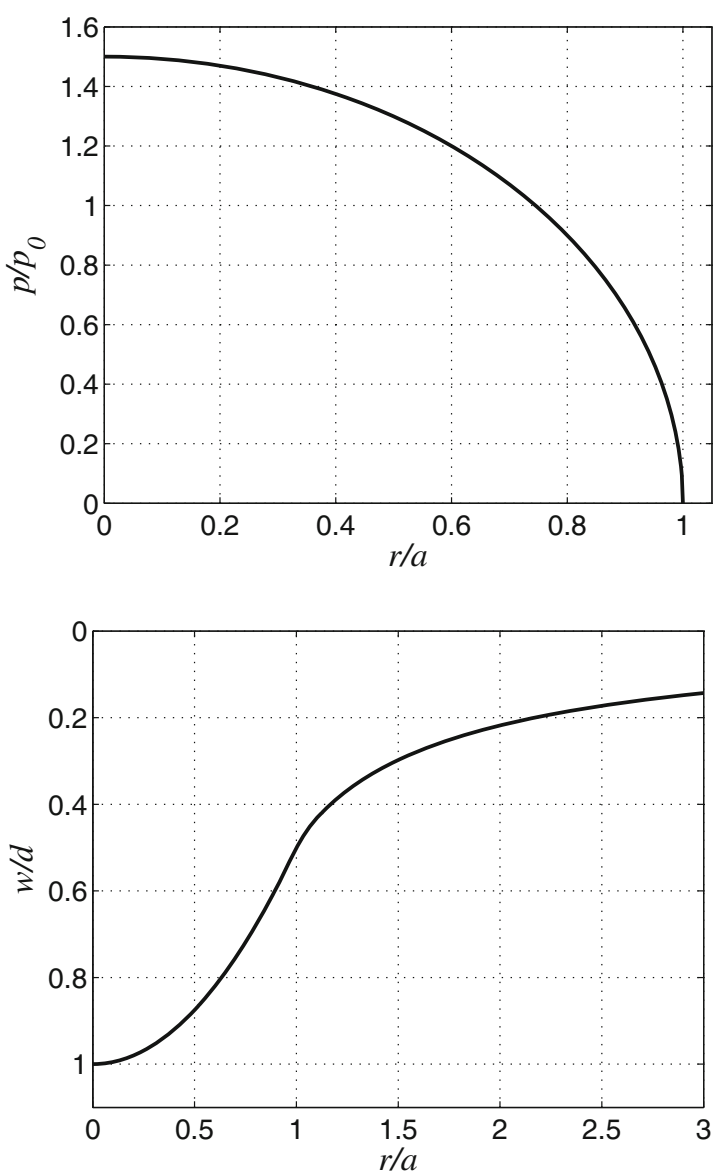

following solution:

$$
\begin{aligned}
\frac{\sigma_{r r}(r, z ; a)}{p_{0}}= & \frac{3}{2}\left\{\frac{1-2 v}{3} \frac{a^{2}}{r^{2}}\left[1-\left(\frac{z}{\sqrt{u}}\right)^{3}\right]+\left(\frac{z}{\sqrt{u}}\right)^{3} \frac{a^{2} u}{u^{2}+a^{2} z^{2}}\right. \\
& \left.+\frac{z}{\sqrt{u}}\left[\frac{(1-v) u}{a^{2}+u}+(1+v) \frac{\sqrt{u}}{a} \arctan \left(\frac{a}{\sqrt{u}}\right)-2\right]\right\}, \\
\frac{\sigma_{\varphi \varphi}(r, z ; a)}{p_{0}}= & -\frac{3}{2}\left\{\frac{1-2 v}{3} \frac{a^{2}}{r^{2}}\left[1-\left(\frac{z}{\sqrt{u}}\right)^{3}\right]\right. \\
& \left.+\frac{z}{\sqrt{u}}\left[2 v+\frac{(1-v) u}{a^{2}+u}-(1+v) \frac{\sqrt{u}}{a} \arctan \left(\frac{a}{\sqrt{u}}\right)\right]\right\},
\end{aligned}
$$




$$
\begin{aligned}
\frac{\sigma_{z z}(r, z ; a)}{p_{0}} & =-\frac{3}{2}\left(\frac{z}{\sqrt{u}}\right)^{3} \frac{a^{2} u}{u^{2}+a^{2} z^{2}}, \\
\frac{\sigma_{r z}(r, z ; a)}{p_{0}} & =-\frac{3}{2} \frac{r z^{2}}{u^{2}+a^{2} z^{2}} \frac{a^{2} \sqrt{u}}{u+a^{2}},
\end{aligned}
$$

with the Poisson's ratio $v$ and the expression:

$$
u(r, z ; a)=\frac{1}{2}\left(r^{2}+z^{2}-a^{2}+\sqrt{\left(r^{2}+z^{2}-a^{2}\right)^{2}+4 a^{2} z^{2}}\right) .
$$

The stresses $\sigma_{r \varphi}$ and $\sigma_{\varphi z}$ vanish due to rotational symmetry. Figure 2.13 displays the von-Mises equivalent stress-normalized to the average pressure in the contactresulting from this stress tensor. It is apparent that the equivalent stress reaches its greatest value in the middle of contact, yet underneath the half-space surface. Therefore, the parabolic indenter is not suitable for measuring hardness as Hertz (1882) had originally assumed. Figure 2.14 displays the greatest resulting principle stresses in the half-space.

An alternative, yet naturally fully equivalent formulation of the stresses in the half-space given by (2.32), can be referenced within work by Hamilton and Goodman (1966).

\section{The Hertzian Impact Problem}

Hertz (1882) studied the impact problem for this contact also. Consider the parabolic indenter of mass $m$ normally impacting the initially non-deformed halfspace with the initial speed $v_{0}$. Let the impact be quasi-static, i.e., $v_{0} \ll c$, where $c$ represents the characteristic propagation speed of elastic waves in the half-space. The energy radiation in the form of elastic waves in the half-space can then be neglected, as demonstrated by Hunter (1957). In (2.29) the relationship between

Fig. 2.13 Von-Mises equivalent stress curve, normalized to the average pressure in the half-space for the indentation by a paraboloid assuming $\nu=0.3$

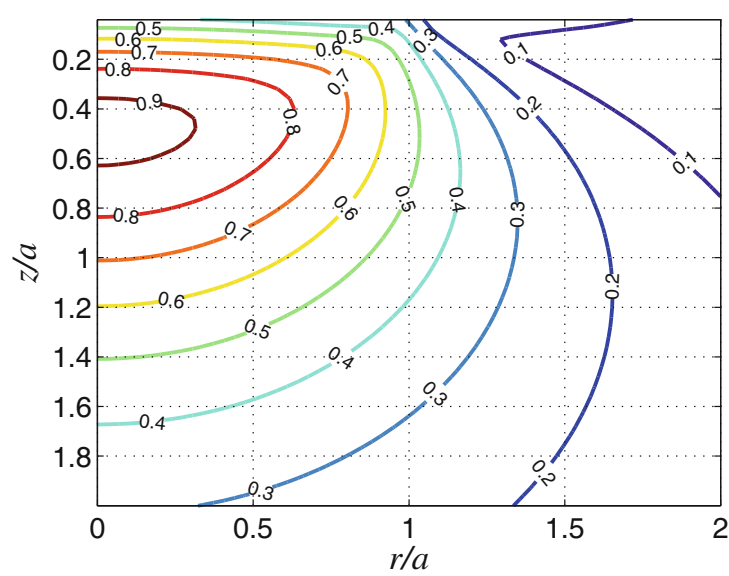


Fig. 2.14 Greatest principle stress curve, normalized to the average pressure in the half-space for the indentation by a paraboloid assuming $v=0.3$

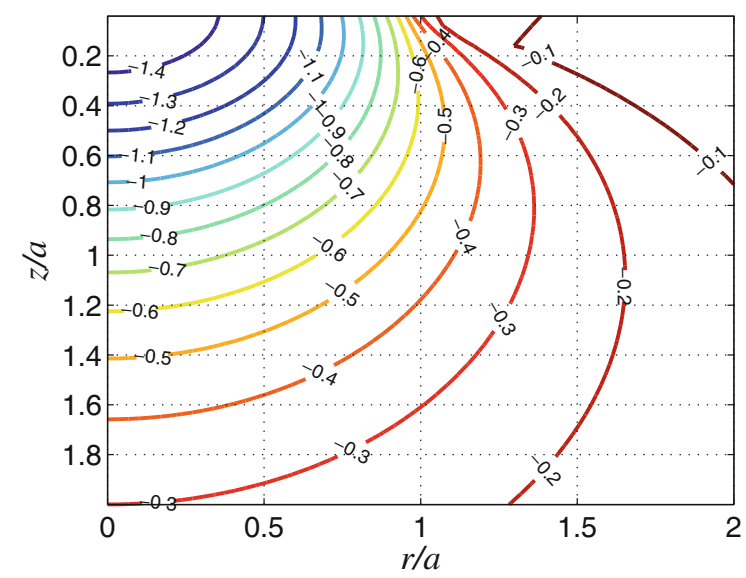

normal force $F_{N}$ and indentation depth $d$ is implicitly given:

$$
F_{N}(d)=\frac{4}{3} E^{*} \sqrt{R d^{3}}
$$

Therefore, the potential energy stored in the elastic deformation of the elastic halfspace, $U$, is given by:

$$
U(d)=\int_{0}^{d} F(\tilde{d}) \mathrm{d} \tilde{d}=\frac{8}{15} E^{*} \sqrt{R d^{5}}
$$

The indentation depth during the impact is a function of time, $d=d(t)$, and for the quasi-static case the energy conservation during impact takes on the following simple form:

$$
m \frac{v_{0}^{2}}{2}=m \frac{\dot{d}^{2}}{2}+\frac{8}{15} E^{*} \sqrt{R d^{5}} .
$$

This equation yields the maximum indentation depth $d_{\max }$, the function $t=t(d)$, i.e., the inverse of the time dependence of the indentation depth $d=d(t)$, and the impact duration $t_{S}$ :

$$
\begin{aligned}
d_{\max } & =\left(\frac{15 m v_{0}^{2}}{16 E^{*} \sqrt{R}}\right)^{2 / 5}, \\
t & =\frac{2}{5} \frac{d_{\max }}{v_{0}} \mathrm{~B}\left(\xi ; \frac{2}{5}, \frac{1}{2}\right), \quad \xi=\left(\frac{d}{d_{\max }}\right)^{5 / 2} \\
t_{S} & =\frac{4}{5} \frac{d_{\max }}{v_{0}} \mathrm{~B}\left(1 ; \frac{2}{5}, \frac{1}{2}\right) \approx 2.94 \frac{d_{\max }}{v_{0}} .
\end{aligned}
$$


Here, $\mathrm{B}(\cdot ; \cdot, \cdot)$ is the incomplete beta function

$$
\mathrm{B}(z ; a, b):=\int_{0}^{z} t^{a-1}(1-t)^{b-1} \mathrm{~d} t .
$$

\subsubsection{The Sphere}

The problem of the spherical indenter is very closely related to the problem of the parabolic indenter described in the previous section. The profile of a sphere of radius $R$ is:

$$
f(r)=R-\sqrt{R^{2}-r^{2}} .
$$

For the case of $r \ll R$ this can be approximated as:

$$
f(r) \approx \frac{r^{2}}{2 R},
$$

which obviously coincides with (2.28) from Sect. 2.5.3. It may now be necessary to use the exact spherical shape instead of the parabolic approximation. However, the assumption of small deformations that underlies the whole theory used in this book requires the validity of the half-space hypothesis, which in our case can be written as $a \ll R$. If the latter is fulfilled, one can still work with the parabolic approximation. Nevertheless, we want to present the solution of the contact problem with a spherical indenter, which was first published by Segedin (1957). Applying (2.17) to (2.39) yields:

$$
\begin{gathered}
g(x)|x| \int_{0}^{|x|} \frac{r \mathrm{~d} r}{\sqrt{R^{2}-r^{2}} \sqrt{x^{2}-r^{2}}}=|x| \operatorname{artanh}\left(\frac{|x|}{R}\right), \\
d(a)=a \operatorname{artanh}\left(\frac{a}{R}\right), \\
F_{N}(a)=2 E^{*} \int_{0}^{a}\left[a \operatorname{artanh}\left(\frac{a}{R}\right)-x \operatorname{artanh}\left(\frac{x}{R}\right)\right] \mathrm{d} x \\
=E^{*} R^{2}\left[\left(1+\frac{a^{2}}{R^{2}}\right) \operatorname{artanh}\left(\frac{a}{R}\right)-\frac{a}{R}\right] .
\end{gathered}
$$

Here $\operatorname{artanh}(\cdot) \operatorname{refers}$ to the area hyperbolic tangent function, which can also be represented explicitly by the natural logarithm:

$$
\operatorname{artanh}\left(\frac{a}{R}\right)=\frac{1}{2} \ln \left(\frac{R+a}{R-a}\right) .
$$


The average pressure in the contact is:

$$
p_{0}=\frac{E^{*} R^{2}}{\pi a^{2}}\left[\left(1+\frac{a^{2}}{R^{2}}\right) \operatorname{artanh}\left(\frac{a}{R}\right)-\frac{a}{R}\right] .
$$

The stresses and displacements were not determined by Segedin (1957), and can only be partially expressed by elementary functions. With the help of (2.17) we obtain the relationships:

$$
\begin{aligned}
& \sigma_{z z}(r ; a)=-\frac{E^{*}}{\pi} \int_{r}^{a}\left[\frac{x R}{R^{2}-x^{2}}+\operatorname{artanh}\left(\frac{x}{R}\right)\right] \frac{\mathrm{d} x}{\sqrt{x^{2}-r^{2}}}, \quad r \leq a, \\
& =-\frac{E^{*}}{\pi}\left[\frac{R}{\sqrt{R^{2}-r^{2}}} \operatorname{artanh}\left(\frac{\sqrt{a^{2}-r^{2}}}{\sqrt{R^{2}-r^{2}}}\right)\right. \\
& \left.+\int_{r}^{a} \operatorname{artanh}\left(\frac{x}{R}\right) \frac{\mathrm{d} x}{\sqrt{x^{2}-r^{2}}}\right] \\
& w(r ; a)=\frac{2}{\pi}\left[a \operatorname{artanh}\left(\frac{a}{R}\right) \arcsin \left(\frac{a}{r}\right)-\int_{0}^{a} x \operatorname{artanh}\left(\frac{x}{R}\right) \frac{\mathrm{d} x}{\sqrt{r^{2}-x^{2}}}\right], \\
& r>a, \\
& =\frac{2}{\pi}\left\{\operatorname{artanh}\left(\frac{a}{R}\right)\left[\operatorname{a} \arcsin \left(\frac{a}{r}\right)+\sqrt{r^{2}-a^{2}}\right]-R \arcsin \left(\frac{a}{r}\right)\right. \\
& \left.+\sqrt{R^{2}-r^{2}} \arctan \left(\frac{a \sqrt{R^{2}-r^{2}}}{R \sqrt{r^{2}-a^{2}}}\right)\right\} \text {. }
\end{aligned}
$$

These functions are shown in normalized form in Figs. 2.15 and 2.16 for different values of $R / a$. It can be seen that, even for small values of this ratio such as 1.5 (which already strongly violates the half-space approximation), the stresses are only slightly different and the displacements almost indistinguishable from the parabolic approximation in Sect. 2.5.3.

\subsubsection{The Ellipsoid}

The solution for an indenter in the form of an ellipsoid of rotation also originated from Segedin (1957). The profile is given by:

$$
f(r)=R\left(1-\sqrt{1-k^{2} r^{2}}\right)
$$


Fig. 2.15 Normal pressure $p=-\sigma_{z z}$, normalized to the average pressure in the contact, $p_{0}$, for indentation by a sphere for different ratios $R / a$. The thin solid line indicates the parabolic approximation from (2.30)

Fig. 2.16 Displacement of the half-space, normalized to the indentation depth $d$ for indentation by a sphere for different ratios $R / a$. Since the curves are approximately on top of each other, the line of the parabolic approximation, according to (2.30), has been omitted
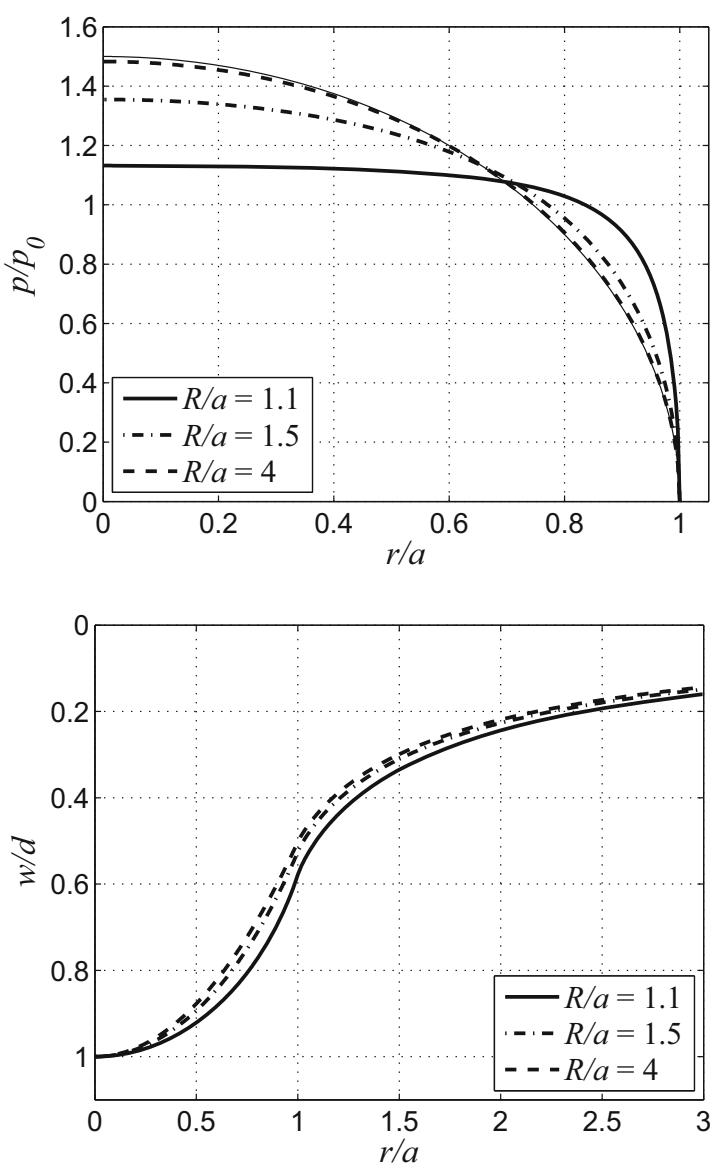

with the two parameters, $R$ and $k . k R=1$, resulting in the spherical indenter of the previous section. In general cases, the equivalent profile is as follows:

$$
\begin{aligned}
g(x) & =|x| R \int_{0}^{|x|} \frac{k^{2} r \mathrm{~d} r}{\sqrt{1-k^{2} r^{2}} \sqrt{x^{2}-r^{2}}}=|x| k R \operatorname{artanh}(k|x|) \\
& =k R g_{\text {sphere }}\left(x ; R=\frac{1}{k}\right) .
\end{aligned}
$$

Here, $g_{\text {sphere }}(x ; R)$ denotes the solution:

$$
g_{\text {sphere }}(x ; R):=|x| \operatorname{artanh}\left(\frac{|x|}{R}\right)
$$

derived in the previous section for a sphere with the radius $R$. Because of the superposition principle, all expressions for the stresses and displacements-and, 
correspondingly of course, also for the macroscopic quantities-are linear in $g$. Therefore, it is clear without calculation that the solution of the contact problem is given by:

$$
\begin{aligned}
d(a) & =k R d_{\text {sphere }}\left(a ; R=\frac{1}{k}\right), \\
F_{N}(a) & =k R F_{N \text {,sphere }}\left(a ; R=\frac{1}{k}\right), \\
\sigma_{z z}(r ; a) & =k R \sigma_{z z \text {,sphere }}\left(r ; a ; R=\frac{1}{k}\right), \quad r \leq a, \\
w(r ; a) & =k R w_{\text {sphere }}\left(r ; a ; R=\frac{1}{k}\right), \quad r>a .
\end{aligned}
$$

The index "sphere" denotes the respective solution from Sect. 2.5.4.

\subsubsection{The Profile Which Generates Constant Pressure}

It is possible to design an indenter in such a way that the generated pressure in the contact is constant. This contact problem was initially solved by Lamb (1902) in the form of hypergeometric functions and by utilizing the potentials of Boussinesq. We will present a slightly simplified solution based on elliptical integrals, which goes back to Föppl (1941).

Applying a constant pressure $p_{0}$ to a circular region with the radius $a$ yields the following vertical displacements $w_{1 D}(x)$ in a one-dimensional MDR model according to (2.16):

$$
w_{1 D}(x)=\frac{2}{E^{*}} \int_{x}^{a} \frac{r p_{0}}{\sqrt{r^{2}-x^{2}}} \mathrm{~d} r=\frac{2 p_{0}}{E^{*}} \sqrt{a^{2}-x^{2}} .
$$

The displacement in the real three-dimensional space is given by:

$$
\begin{aligned}
w(r) & =\frac{2}{\pi} \int_{0}^{r} \frac{w_{1 D}(x)}{\sqrt{r^{2}-x^{2}}} \mathrm{~d} x=\frac{4 p_{0}}{\pi E^{*}} \int_{0}^{r} \frac{\sqrt{a^{2}-x^{2}}}{\sqrt{r^{2}-x^{2}}} \mathrm{~d} x \\
& =\frac{4 p_{0} a}{\pi E^{*}} \mathrm{E}\left(\frac{r}{a}\right), \quad r \leq a .
\end{aligned}
$$

Here, $E(\cdot)$ denotes the complete elliptical integral of the second kind:

$$
\mathrm{E}(k):=\int_{0}^{\pi / 2} \sqrt{1-k^{2} \sin ^{2} \varphi} \mathrm{d} \varphi .
$$


The indentation depth $d$ is therefore:

$$
d=w(0)=\frac{2 p_{0} a}{E^{*}}
$$

and the shape of the profile is given by:

$$
f(r)=d-w(r)=\frac{2 p_{0} a}{E^{*}}\left[1-\frac{2}{\pi} \mathrm{E}\left(\frac{r}{a}\right)\right] .
$$

It is apparent that this is not a classical indenter with a constant shape: varying $p_{0}$ causes the profile to be scaled. In other words, different pairings $\left\{a, p_{0}\right\}$ require different indenter profiles $f(r)$. Concrete applications, usually in biological systems, are discovered upon considering the adhesive normal contact. We will examine them at a later point. For completeness, we will calculate the displacement outside the contact area:

$$
\begin{aligned}
w\left(r ; a, p_{0}\right) & =\frac{4 p_{0}}{\pi E^{*}} \int_{0}^{a} \frac{\sqrt{a^{2}-x^{2}} \mathrm{~d} x}{\sqrt{r^{2}-x^{2}}}, \quad r>a, \\
& =\frac{4 p_{0} r}{\pi E^{*}}\left[\mathrm{E}\left(\frac{a}{r}\right)-\left(1-\frac{a^{2}}{r^{2}}\right) \mathrm{K}\left(\frac{a}{r}\right)\right],
\end{aligned}
$$

with the complete elliptical integral of the first kind:

$$
\mathrm{K}(k):=\int_{0}^{\pi / 2} \frac{\mathrm{d} \varphi}{\sqrt{1-k^{2} \sin ^{2} \varphi}} .
$$

The displacement $w$ of the half-space is shown in Fig. 2.17.

Fig. 2.17 Displacements within and outside the contact area, normalized to the indentation depth, for an indenter generating constant pressure

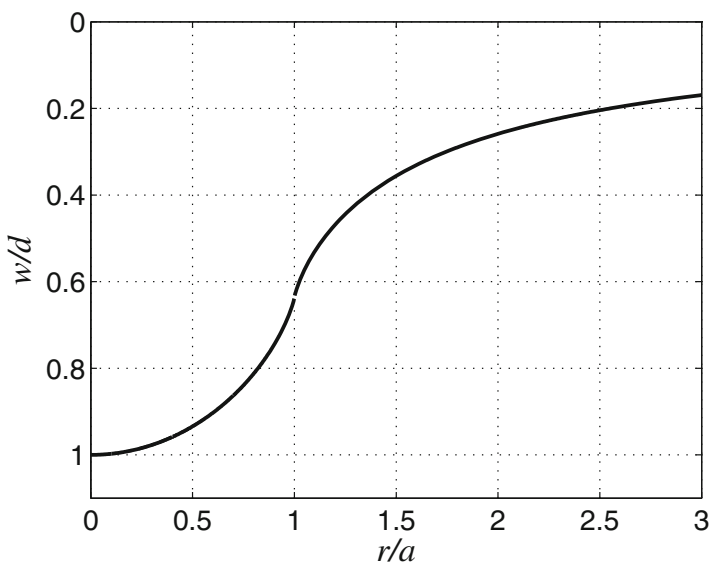


Fig. 2.18 Thin circular ring. Visualized derivation of the integral (2.58)

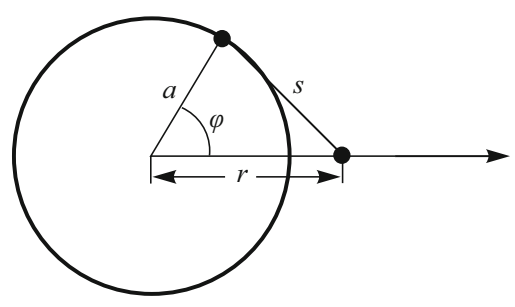

\subsubsection{Displacement from Indentation by a Thin Circular Ring}

We now examine the indentation of the elastic half-space by a thin circular ring of radius $a$. Let the ring be sufficiently thin so that the pressure distribution can be regarded as a Dirac distribution:

$$
\sigma_{z z}(r ; a)=-\frac{F_{N}}{2 \pi a} \delta(r-a)
$$

where $F_{N}$ denotes the normal force loading the ring. The resulting displacement of the half-space can be determined from the superposition of fundamental solutions of elasticity theory. The half-space normal displacement resulting from the point force acting on the origin in the $z$-direction is, according to (2.2), given by:

$$
w(s)=\frac{F_{z}}{\pi E^{*} s},
$$

with the distance $s$ to the acting point of the force.

The displacements (see notations used in the diagram in Fig. 2.18) produced by this pressure distribution (2.56) are given by:

$$
\begin{aligned}
w(r ; a) & =\frac{1}{\pi E^{*}} \int_{0}^{2 \pi} \frac{F_{N}}{2 \pi} \frac{\mathrm{d} \varphi}{\sqrt{a^{2}+r^{2}-2 a r \cos \varphi}} \\
& =\frac{F_{N}}{2 E^{*}} \frac{4}{\pi^{2}(r+a)} \mathrm{K}\left(\frac{2 \sqrt{r a}}{r+a}\right) .
\end{aligned}
$$

These displacements are represented in Fig. 2.19. A superposition of the displacements enables the direct calculation of the displacements from any given axially symmetric pressure distribution.

\subsubsection{The Profile in the Form of a Power-Law}

For a general indenter with the profile in the form of a power-law (see Fig. 2.20),

$$
f(r)=c r^{n}, \quad n \in \mathbb{R}^{+},
$$


Fig.2.19 Normalized surface displacement from indentation by a thin circular ring

Fig. 2.20 Normal indentation by a mnemonic indenter
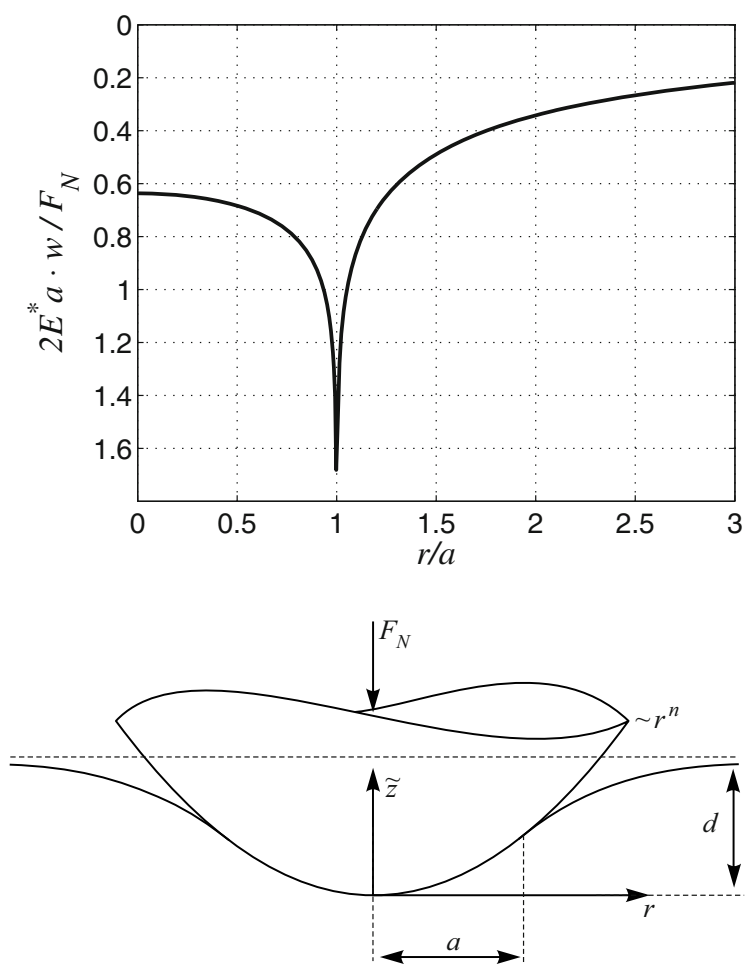

the solution of the contact problem can also be given in explicit form. Here, $c$ is a constant and $n$ is a positive real number. For example, for $n=1$ and $n=2$ arise the already considered cases of a conical or parabolic indenter. The general solution was first found by Galin (1946). Shtaerman (1939) gave a solution in faculty expressions for even integers $n$.

The equivalent plane profile $g(x)$ is, in this case, also a power function with the exponent $n$ :

$$
g(x)=|x| n c \int_{0}^{|x|} \frac{r^{n-1} \mathrm{~d} r}{\sqrt{x^{2}-r^{2}}}=\kappa(n) c|x|^{n},
$$

with the scaling factor

$$
\kappa(n):=\sqrt{\pi} \frac{\Gamma(n / 2+1)}{\Gamma[(n+1) / 2]} .
$$

Here, $\Gamma(\cdot)$ denotes the gamma function

$$
\Gamma(z):=\int_{0}^{\infty} t^{z-1} \exp (-t) \mathrm{d} t
$$


Fig. 2.21 Dependence of the stretch factor $\kappa$ in (2.61) on the exponent $n$ of the power profile

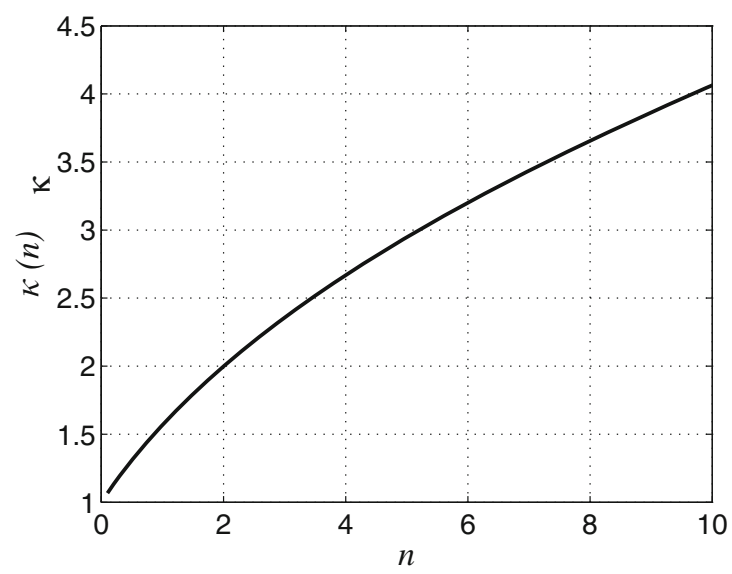

Table 2.1 Scaling factor $\kappa(n)$ for selected exponents of the shape function

\begin{tabular}{l|l|l|l|l|l|l|l|l|l|l|l}
\hline$n$ & 0.5 & 1 & 2 & 3 & 4 & 5 & 6 & 7 & 8 & 9 & 10 \\
\hline$\kappa(n)$ & 1.311 & 1.571 & 2 & 2.356 & 2.667 & 2.945 & 3.2 & 3.436 & 3.657 & 3.866 & 4.063 \\
\hline
\end{tabular}

The dependence of the scaling factor on the exponent $n$ is shown in Fig. 2.21 and in Table 2.1. For the relationships between the normal force $F_{N}$, indentation depth $d$, and contact radius $a$ we get:

$$
\begin{aligned}
d(a) & =\kappa(n) c a^{n}, \\
F_{N}(a) & =E^{*} \frac{2 n}{n+1} \kappa(n) c a^{n+1} .
\end{aligned}
$$

The mean pressure in contact is:

$$
p_{0}=\frac{E^{*}}{\pi} \frac{2 n}{n+1} \kappa(n) c a^{n-1} .
$$

For the stress and displacement distributions, the expressions given in (2.17) will result in:

$$
\begin{aligned}
\sigma_{z z}(r ; a) & =-\frac{E^{*}}{\pi} n \kappa(n) c \int_{r}^{a} x^{n-1} \frac{\mathrm{d} x}{\sqrt{x^{2}-r^{2}}}, \quad r \leq a, \\
& =-\frac{E^{*}}{2 \pi} n \kappa(n) c r^{n-1}\left[\mathrm{~B}\left(1 ; \frac{1-n}{2}, \frac{1}{2}\right)-\mathrm{B}\left(\frac{r^{2}}{a^{2}} ; \frac{1-n}{2}, \frac{1}{2}\right)\right], \\
w(r ; a) & =\frac{2}{\pi} \kappa(n) c\left[a^{n} \arcsin \left(\frac{a}{r}\right)-\int_{0}^{a} x^{n} \frac{\mathrm{d} x}{\sqrt{r^{2}-x^{2}}}\right], \quad r>a, \\
& =\frac{2}{\pi} \kappa(n) c a^{n}\left[\arcsin \left(\frac{a}{r}\right)-\frac{1}{n+1} \frac{a}{r}{ }_{2} \mathrm{~F}_{1}\left(\frac{1}{2}, \frac{n+1}{2} ; \frac{n+3}{2} ; \frac{a^{2}}{r^{2}}\right)\right] .
\end{aligned}
$$


Here, $\mathrm{B}(\cdot ; \cdot, \cdot)$ denotes the incomplete beta function

$$
\mathrm{B}(z ; a, b):=\frac{z^{a}}{a}{ }_{2} \mathrm{~F}_{1}(a, 1-b ; a+1 ; z)
$$

and ${ }_{2} \mathrm{~F}_{1}(\cdot, \cdot ; \cdot ; \cdot)$ the hypergeometric function

$$
{ }_{2} \mathrm{~F}_{1}(a, b ; c ; z):=\sum_{n=0}^{\infty} \frac{\Gamma(a+n) \Gamma(b+n) \Gamma(c)}{\Gamma(a) \Gamma(b) \Gamma(c+n)} \frac{z^{n}}{n !} .
$$

However, the evaluation of these functions is somewhat cumbersome, which is why we give two recursion formulas with which all stresses and displacements for natural values of $n$ can be recursively determined from the known solutions for a cylindrical flat punch and a cone:

$$
\begin{gathered}
\int_{r}^{a} \frac{x^{n-1} \mathrm{~d} x}{\sqrt{x^{2}-r^{2}}}=\frac{a^{n-2} \sqrt{a^{2}-r^{2}}}{n-1}+r^{2} \frac{n-2}{n-1} \int_{r}^{a} \frac{x^{n-3} \mathrm{~d} x}{\sqrt{x^{2}-r^{2}}}, \quad r \leq a, \\
-\int_{0}^{a} \frac{x^{n} \mathrm{~d} x}{\sqrt{r^{2}-x^{2}}}=\frac{a^{n-1} \sqrt{r^{2}-a^{2}}}{n}-r^{2} \frac{n-1}{n} \int_{0}^{a} \frac{x^{n-2} \mathrm{~d} x}{\sqrt{r^{2}-x^{2}}}, \quad r>a .
\end{gathered}
$$

In the case of the cone $(n=1)$, the normal stress has a logarithmic singularity at the apex of the cone. For all $n>1$ the pressure distribution is not singular; the pressure maximum remains at the center of the contact until $n=2$ and then begins to shift to the edge of the contact at higher $n$. In the limiting case $n \rightarrow \infty$ corresponding to a cylindrical flat punch, the pressure distribution is singular at the contact edge.

\section{The Impact Problem for the Indenter with Power-Law Profile}

Since the relationships between normal force, contact radius, and indentation depth are known to be power functions, the normal impact problem can also be solved easily for this indenter profile. Consider a rotationally symmetric rigid body of mass $m$ with the power-law profile just described, which impacts on the elastic half-space with the initial velocity $v_{0}$. The collision should be quasi-static, i.e., this impact velocity would be much smaller than the propagation speed of elastic waves.

The potential energy as a function of the current indentation depth can be derived from (2.63) and is given by:

$$
U(d)=\int_{0}^{d} F(\tilde{d}) \mathrm{d} \tilde{d}=\frac{E^{*}}{[c \kappa(n)]^{1 / n}} \frac{2 n^{2}}{(2 n+1)(n+1)} d^{\frac{2 n+1}{n}} .
$$


Due to energy conservation, the following expressions for the maximum indentation depth $d_{\max }$, the function $t=t(d)$, and the impact duration $t_{S}$ are valid:

$$
\begin{aligned}
d_{\max } & =\left\{\frac{[c \kappa(n)]^{1 / n}(2 n+1)(n+1) m v_{0}^{2}}{4 n^{2} E^{*}}\right\}^{\frac{n}{2 n+1}}, \\
t & =\frac{n}{2 n+1} \frac{d_{\max }}{v_{0}} \mathrm{~B}\left(\xi ; \frac{n}{2 n+1}, \frac{1}{2}\right), \xi=\left(\frac{d}{d_{\max }}\right)^{\frac{2 n+1}{n}}, \\
t_{S} & =\frac{2 n}{2 n+1} \frac{d_{\max }}{v_{0}} \mathrm{~B}\left(1 ; \frac{n}{2 n+1}, \frac{1}{2}\right),
\end{aligned}
$$

which, for $n=2$, coincides with the solution of the Hertzian impact problem described in Sect. 2.5.3.

\subsubsection{The Truncated Cone}

For certain technical applications it can be of interest to consider an indenter with a flattened tip, perhaps worn down by wear. The solution for the indentation by a truncated cone was first published by Ejike (1969). This solution-as well as the general solution by Sneddon (1965) — is based on appropriate integral transforms. A simple solution in the framework of MDR has been provided in this section.

The axisymmetric profile (see Fig. 2.22) can be written as:

$$
f(r)= \begin{cases}0, & r \leq b \\ (r-b) \tan \theta, & r>b .\end{cases}
$$

Here, $\theta$ denotes the slope angle of the cone and $b$ the radius of the blunt end. As in previous cases, the problem can be dealt with elementarily by utilizing (2.17),

Fig.2.22 Normal indentation by a truncated cone

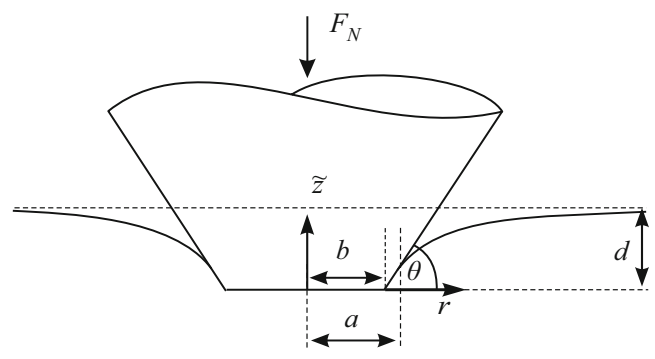


which provide the solution:

$$
\begin{aligned}
g(x) & = \begin{cases}0, & |x| \leq b, \\
|x| \tan \theta \arccos \left(\frac{b}{|x|}\right), & |x|>b,\end{cases} \\
d(a) & =a \tan \theta \arccos \left(\frac{b}{a}\right)=\varphi_{0} a \tan \theta, \\
F_{N}(a) & =E^{*} \tan \theta a^{2}\left[\arccos \left(\frac{b}{a}\right)+\frac{b}{a} \sqrt{1-\frac{b^{2}}{a^{2}}}\right] \\
& =E^{*} \tan \theta a^{2}\left(\varphi_{0}+\cos \varphi_{0} \sin \varphi_{0}\right),
\end{aligned}
$$

where we introduced the angle

$$
\varphi_{0}:=\arccos \left(\frac{b}{a}\right) .
$$

The average pressure in the contact is:

$$
p_{0}=\frac{E^{*}}{\pi} \tan \theta\left(\varphi_{0}+\cos \varphi_{0} \sin \varphi_{0}\right) .
$$

Setting $b=0$, i.e., $\varphi_{0}=\pi / 2$, yields the solution of the ideal conical indenter from Sect. 2.5.2. For the stresses in the contact area we obtain:

$$
\begin{aligned}
& \sigma_{z z}(r ; a)= \\
& -\frac{E^{*} \tan \theta}{\pi}\left\{\begin{array}{l}
\int_{b}^{a}\left\{\frac{b}{\sqrt{x^{2}-b^{2}}}+\arccos \left(\frac{b}{x}\right)\right\} \frac{\mathrm{d} x}{\sqrt{x^{2}-r^{2}}}, \quad r \leq b, \\
\int_{r}^{a}\left\{\frac{b}{\sqrt{x^{2}-b^{2}}}+\arccos \left(\frac{b}{x}\right)\right\} \frac{\mathrm{d} x}{\sqrt{x^{2}-r^{2}}}, \quad b<r \leq a .
\end{array}\right.
\end{aligned}
$$

The stresses are shown in Fig. 2.23. The singularity at the sharp edge of the blunt end, $r=b$, is clearly visible. For small values of $b / a$, the curves approach the solution for the complete cone, and for $b / a \rightarrow 1$ the limiting case of the flat cylindrical punch. Both limiting cases are represented as thin solid lines in Figs. 2.23 and 2.24. The displacements outside of the contact area are:

$$
\begin{gathered}
w(r ; a)=\frac{2 \tan \theta}{\pi}\left\{\varphi_{0} a \arcsin \left(\frac{a}{r}\right)-\int_{b}^{a} x \arccos \left(\frac{b}{x}\right) \frac{\mathrm{d} x}{\sqrt{r^{2}-x^{2}}}\right\}, \\
r>a .
\end{gathered}
$$


Fig. 2.23 Stress distribution, normalized to the average pressure in the contact, for the indentation by a truncated cone with different values $b / a$. The thin solid lines represent the solutions for the complete cone and the flat cylindrical punch

Fig. 2.24 Displacements of the half-space, normalized to the indentation depth, for the indentation by a truncated cone with different values $b / a$. The thin solid lines represent the solutions for the complete cone and the flat cylindrical punch
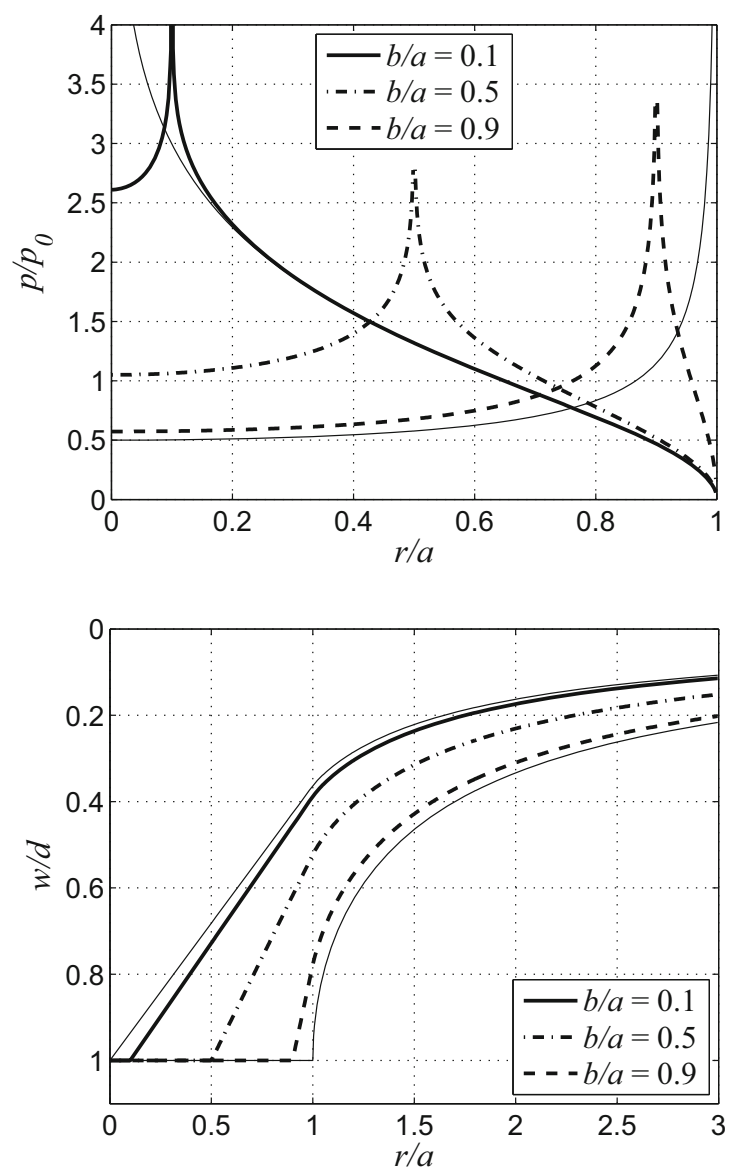

Theoretically, this integral can be solved analytically using tabulated functions, but due to its complexity, a numerical solution seems more feasible. These displacements are displayed in Fig. 2.24.

\subsubsection{The Truncated Paraboloid}

The indentation problem for a truncated paraboloid (see Fig. 2.25) was also first solved by Ejike (1981). The profile is described by:

$$
f(r)= \begin{cases}0, & r \leq b, \\ \frac{r^{2}-b^{2}}{2 R}, & r>b,\end{cases}
$$


Fig. 2.25 Normal indentation by a truncated paraboloid

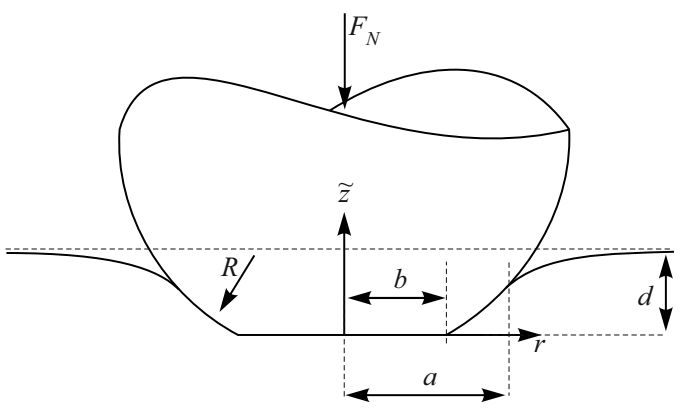

with the radius of the blunt end, $b$, and the radius of curvature of the paraboloid $R$. The solution within the MDR (see (2.17)) gives:

$$
\begin{aligned}
g(x) & = \begin{cases}0, & |x| \leq b, \\
\frac{|x|}{R} \sqrt{x^{2}-b^{2}}, & |x|>b,\end{cases} \\
d(a) & =\frac{a}{R} \sqrt{a^{2}-b^{2}}, \\
F_{N}(a) & =\frac{2 E^{*}}{3 R}\left(2 a^{2}+b^{2}\right) \sqrt{a^{2}-b^{2}} .
\end{aligned}
$$

For the stresses within the contact area, we obtain:

$$
\sigma_{z z}(r ; a)=-\frac{E^{*}}{\pi R} \begin{cases}\int_{b}^{a} \frac{\left(2 x^{2}-b^{2}\right) \mathrm{d} x}{\sqrt{x^{2}-b^{2}} \sqrt{x^{2}-r^{2}}}, & r \leq b, \\ \int_{r}^{a} \frac{\left(2 x^{2}-b^{2}\right) \mathrm{d} x}{\sqrt{x^{2}-b^{2}} \sqrt{x^{2}-r^{2}}}, & b<r \leq a .\end{cases}
$$

These can theoretically be expressed in closed form via elliptic integrals. However, the resulting expressions are very extensive and cumbersome to evaluate, which is why a numerical evaluation will be preferable. The stresses are shown in Fig. 2.26. One recognizes the singularities at the sharp edge of the blunt end at $r=b$, which are increasingly localized for small values of $b$, and the limiting cases of the paraboloid and the cylindrical flat punch (indicated by thin solid lines). The displacements outside the contact area are as follows:

$$
\begin{aligned}
w(r ; a)= & \frac{2 a}{\pi R} \sqrt{a^{2}-b^{2}} \arcsin \left(\frac{a}{r}\right) \\
- & \frac{1}{\pi R}\left[\left(r^{2}-b^{2}\right) \arcsin \left(\frac{\sqrt{a^{2}-b^{2}}}{\sqrt{r^{2}-b^{2}}}\right)-\sqrt{a^{2}-b^{2}} \sqrt{r^{2}-a^{2}}\right], \\
r & >a .
\end{aligned}
$$

These are shown in Fig. 2.27. For $b=0$ we obtain the Hertzian solutions for the parabolic indenter given in Sect. 2.5.3. 
Fig. 2.26 Stress distribution, normalized to the mean pressure in contact, for indentation by a flattened paraboloid at different values $b / a$. The thin solid lines indicate the solutions for the complete paraboloid and the flat punch

Fig. 2.27 Displacements of the half-space, normalized to the indentation depth, for indentation by a flattened paraboloid at different values $b / a$. The thin solid lines indicate the solutions for the complete paraboloid and the flat punch
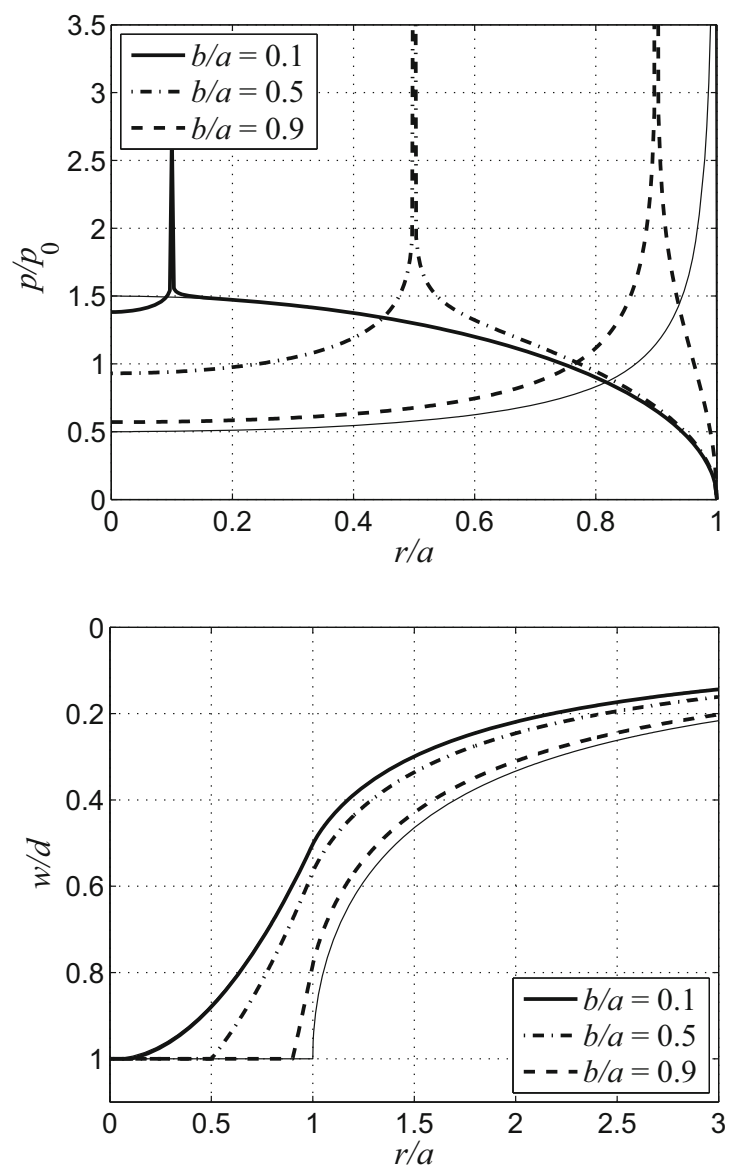

\subsubsection{The Cylindrical Flat Punch with Parabolic Cap}

For mechanical microscopy and other metrological applications, indenters with a parabolic cap are important. In the following, we examine such indenters, assuming that the indentation depth is large enough to ensure that the face comes into complete contact (otherwise we would deal with the simple parabolic contact studied in Sect. 2.5.3).

The solution for a flat cylindrical punch (as a base body) stems from Abramian et al. (1964). In their solution the authors used series expansions to fulfill the field equations. We again show the easier solution within the framework of MDR. The indenter has the profile (see Fig. 2.28)

$$
f(r)= \begin{cases}\frac{r^{2}}{2 R}, & r \leq a, \\ \infty, & r>a,\end{cases}
$$


Fig. 2.28 Normal indentation by a cylindrical punch with parabolic cap

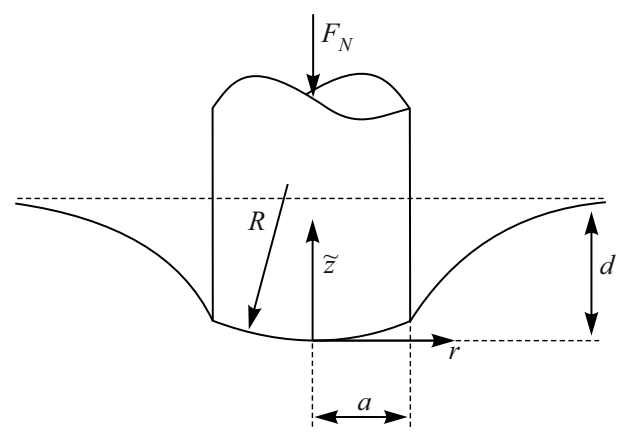

with the radius of curvature of the cap $R$, and the radius of the base punch $a$; the solution of the contact problem is, according to (2.17), given by:

$$
\begin{aligned}
g(x)= & \begin{cases}\frac{x^{2}}{R}, & |x| \leq a \\
\infty, & |x|>a\end{cases} \\
F_{N}(d)= & 2 E^{*}\left(d a-\frac{a^{3}}{3 R}\right), \quad d R \geq a^{2}, \\
\sigma_{z z}(r ; d)= & -\frac{E^{*}}{\pi R} \frac{a^{2}-2 r^{2}+d R}{\sqrt{a^{2}-r^{2}}}, \quad r \leq a, d R \geq a^{2}, \\
w(r ; d)= & \frac{1}{\pi R}\left[\left(2 d R-r^{2}\right) \arcsin \left(\frac{a}{r}\right)+a \sqrt{r^{2}-a^{2}}\right], \\
& r>a, d R \geq a^{2} .
\end{aligned}
$$

For $d R=a^{2}$ (of course), we obtain the Hertzian solution from Sect. 2.5.3, and for $R \rightarrow \infty$ that for the cylindrical flat punch from Sect. 2.5.1. The stresses and

Fig. 2.29 Stress distribution, normalized to the mean pressure in contact, for indentation by a flat punch with parabolic cap at different values $d R / a^{2}$. The thin solid lines indicate the solutions for the paraboloid and the flat punch

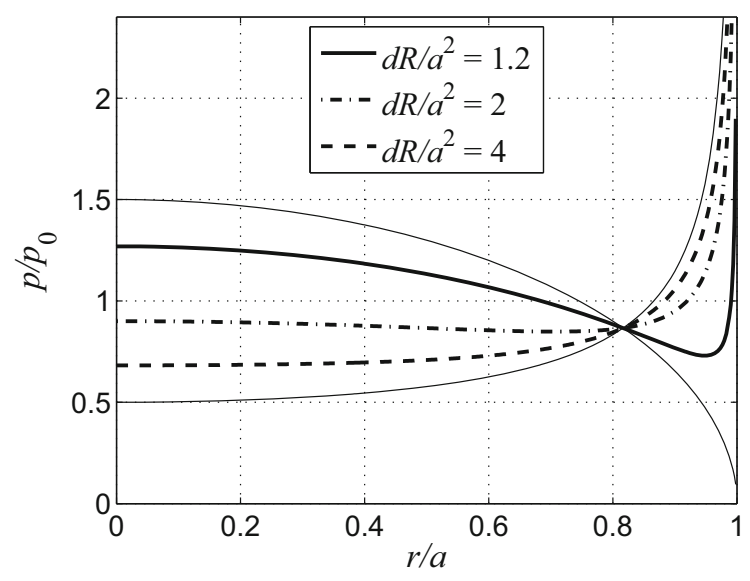


Fig. 2.30 Displacements normalized to the indentation depth, for indentation by a flat punch with parabolic cap at different values $d R / a^{2}$. The thin solid lines indicate the solutions for the paraboloid and the flat punch

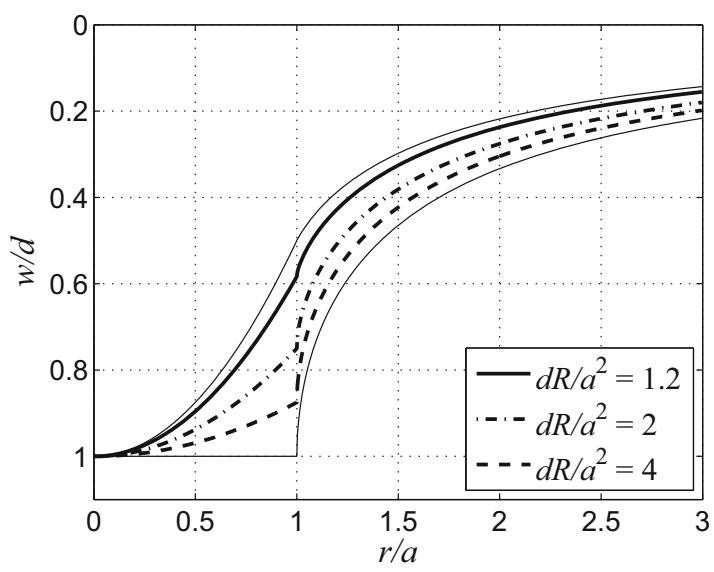

displacements for various values of $d R / a^{2}$ are shown in Figs. 2.29 and 2.30. One recognizes the limiting cases of the paraboloid and the flat punch marked by solid lines. The intersection of all normalized pressure curves is at $r / a=\sqrt{2 / 3}$ and $p / p_{0}=\sqrt{3} / 2$.

\subsubsection{The Cone with Parabolic Cap}

The solution for a cone with a rounded tip, despite its metrological significance, was found first by Ciavarella (1999). However, Maugis and Barquins (1983) already knew the relations between the global contact quantities-normal force, contact radius, and indentation depth-by solving the respective problem with adhesion. Ciavarella's solution is based on the general solution by Shtaerman (1949).

The profile (see Fig. 2.31) is described by:

$$
f(r)= \begin{cases}\frac{r^{2} \tan \theta}{2 b}, & r \leq b, \\ r \tan \theta-\frac{b}{2} \tan \theta, & r>b,\end{cases}
$$

Fig. 2.31 Normal indentation by a cone with a rounded tip

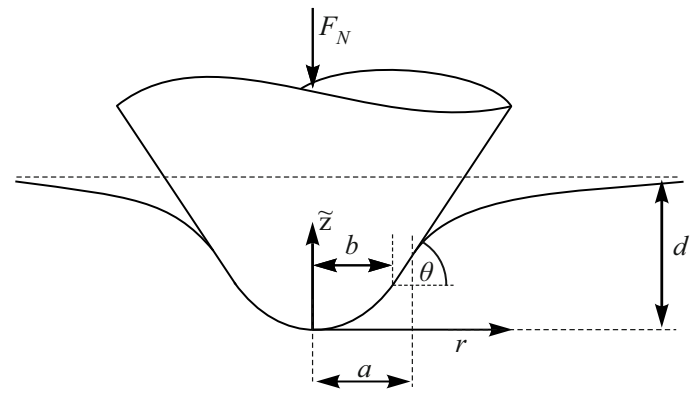


with the radius $b$ at the base of the parabolic cap and the slope angle $\theta$ of the cone. Note that the radius of curvature of the cap is given by $R:=b / \tan \theta$ to guarantee continuous differentiability. For the solution to the contact problem, according to (2.17) one obtains:

$$
\begin{aligned}
g(x) & = \begin{cases}\frac{x^{2} \tan \theta}{b}, & |x| \leq b, \\
\frac{|x| \tan \theta}{b}\left\{|x|-\sqrt{x^{2}-b^{2}}+b \arccos \left(\frac{b}{|x|}\right)\right\}, & |x|>b,\end{cases} \\
d(a) & =\frac{a}{b} \tan \theta\left(a-\sqrt{a^{2}-b^{2}}+b \arccos \left(\frac{b}{a}\right)\right) \\
& =a \tan \theta\left(\frac{1-\sin \varphi_{0}}{\cos \varphi_{0}}+\varphi_{0}\right), \\
F_{N}(a) & =\frac{E^{*} a^{2} \tan \theta}{b}\left[b \arccos \left(\frac{b}{a}\right)+\frac{4}{3}\left(a-\sqrt{a^{2}-b^{2}}\right)+\frac{1}{3} \frac{b^{2} \sqrt{a^{2}-b^{2}}}{a^{2}}\right] \\
& =E^{*} a^{2} \tan \theta\left(\varphi_{0}+\frac{4}{3} \frac{1-\sin \varphi_{0}}{\cos \varphi_{0}}+\frac{1}{3} \sin \varphi_{0} \cos \varphi_{0}\right),
\end{aligned}
$$

where we introduced the angle:

$$
\varphi_{0}:=\arccos \left(\frac{b}{a}\right) .
$$

In the limit $b \rightarrow 0$ we obtain the expressions for the conical indenter from Sect. 2.5.2, because

$$
\lim _{\varphi_{0} \rightarrow \pi / 2} \frac{1-\sin \varphi_{0}}{\cos \varphi_{0}}=0 .
$$

For $b \rightarrow a$, i.e., $\varphi_{0}=0$, we obtain solutions of the Hertzian contact problem from Sect. 2.5.3. For the stresses in the contact area the following expressions result:

$$
\begin{aligned}
& \sigma_{z z}(r ; a)=-\frac{E^{*} \tan \theta}{\pi b} \\
& .\left\{\begin{array}{l}
2 \sqrt{a^{2}-r^{2}}+b \int_{0}^{\varphi_{0}} \frac{(\varphi-2 \tan \varphi) \tan \varphi \mathrm{d} \varphi}{\sqrt{1-k^{2} \cos ^{2} \varphi}}, \quad r \leq b, \\
2 \sqrt{a^{2}-r^{2}}+b \int_{0}^{\operatorname{arcosh}(a / r)}\left[\arccos \left(\frac{b}{r \cosh \varphi}\right)-2 \sqrt{k^{2} \cosh ^{2} \varphi-1}\right] \mathrm{d} \varphi, \\
b<r \leq a,
\end{array}\right.
\end{aligned}
$$


Fig. 2.32 Stress distribution, normalized to the mean pressure in contact, for the indentation by a cone with a rounded tip at different values $b / a$. The thin solid lines indicate the solutions for the paraboloid and the cone

Fig. 2.33 Displacements of the half-space, normalized to the indentation depth, for indentation by a cone with a rounded tip at different values $b / a$. The thin solid lines indicate the solutions for the paraboloid and the cone
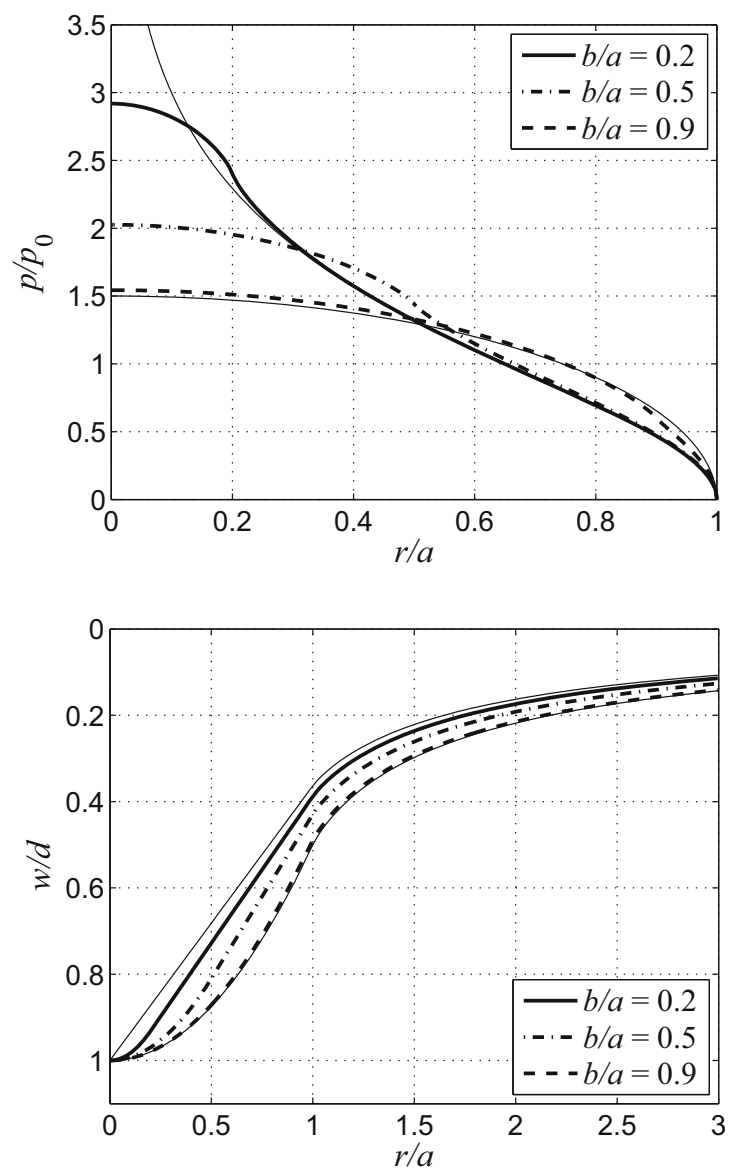

with the parameter $k:=r / b$. For the displacement outside the contact area we get:

$$
\begin{aligned}
w(r ; a) & =\frac{2 d(a)}{\pi} \arcsin \left(\frac{a}{r}\right) \\
-\frac{\tan \theta}{\pi b} & {\left[\begin{array}{c}
\left.r^{2} \arcsin \left(\frac{a}{r}\right)-a \sqrt{r^{2}-a^{2}}+2 b^{2} \int_{0}^{\varphi_{0}} \frac{(\varphi-\tan \varphi) \tan \varphi \mathrm{d} \varphi}{\cos \varphi \sqrt{k^{2} \cos ^{2} \varphi-1}}\right], \\
r>a .
\end{array}\right.}
\end{aligned}
$$

In Figs. 2.32 and 2.33, the normalized stresses and displacements are shown for some values of the ratio $b / a$. It is easy to see the limiting cases of the conical and parabolic indenters marked by the thin lines. The stress distribution is not singular in the origin due to the rounded tip; this is in contrast to the indentation by an ideal cone. 
From the equivalent plane profile $g(x)$ in (2.84) it can be seen that this contact problem is actually a superposition of three previously solved problems, and the solution can therefore be obtained from the sum of the corresponding solutions given in this section. It is indeed

$$
g(x)=g_{P}\left(x ; R=\frac{b}{\tan \theta}\right)+g_{K S}(x)-g_{P S}\left(x ; R=\frac{b}{\tan \theta}\right) .
$$

Here, $g_{P}$ denotes the equivalent profile of a paraboloid of radius $R$ (see Sect. 2.5.3), $g_{K S}(x)$ the equivalent profile of a truncated cone with the inclination angle $\theta$ and the radius $b$ of the flat "tip" (see Sect. 2.5.8), and $g_{P S}$ the equivalent profile of a truncated paraboloid with the radius $R$ and the radius $b$ of the flat "tip" (see Sect. 2.5.10).

\subsubsection{The Paraboloid with Parabolic Cap}

The contact problem of a paraboloid with parabolic cap indenting an elastic halfspace was first solved by Maugis and Barquins (1983) for the adhesive contact. However, the case without adhesion is of course included in this solution. As will be seen, the problem can be considered as a superposition of the indentations by a paraboloid (see Sect. 2.5.3) and a truncated paraboloid (see Sect. 2.5.10), where the weighting factors depend on the two radii of the cap and the paraboloid $R_{1}$ and $R_{2}$. The profile has the shape (see Fig. 2.34)

$$
f(r)= \begin{cases}\frac{r^{2}}{2 R_{1}}, & r \leq b, \\ \frac{r^{2}-h^{2}}{2 R_{2}}, & r>b .\end{cases}
$$

The radius of curvature of the cap, $R_{1}$, must be greater than that of the base paraboloid, $R_{2}$, to ensure a compact contact area. The continuity of the profile also requires

$$
h^{2}=b^{2}\left(1-\frac{R_{2}}{R_{1}}\right) \text {, }
$$

Fig. 2.34 Normal indentation by a sphere with spherical cap

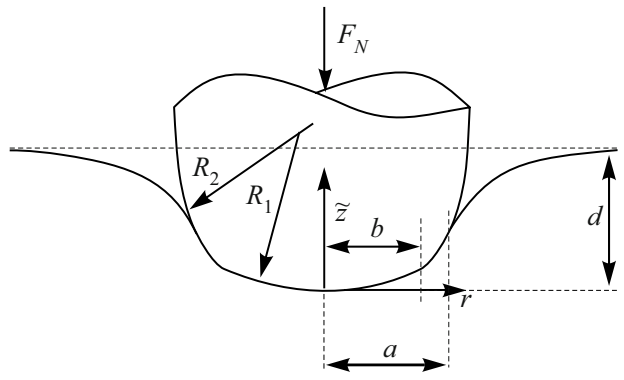


for the length $h$. The equivalent profile within the MDR, seen in (2.17), is given by:

$$
g(x)= \begin{cases}\frac{x^{2}}{R_{1}}, & |x| \leq b, \\ \frac{x^{2}}{R_{1}}+\frac{|x|}{R^{*}} \sqrt{x^{2}-b^{2}}, & |x|>b,\end{cases}
$$

with the effective radius of curvature

$$
R^{*}:=\frac{R_{1} R_{2}}{R_{1}-R_{2}} .
$$

It is easy to see that (2.92) can be thought of as a sum:

$$
g(x)=g_{P}\left(x ; R=R_{1}\right)+g_{P S}\left(x ; R=R^{*}\right) .
$$

Here, $g_{P}$ and $g_{P S}$ denote the equivalent profiles of the paraboloid (see (2.29)) and the truncated paraboloid (see (2.78)). The solution of the contact problem is, therefore, given by:

$$
\begin{aligned}
d(a) & =\frac{a^{2}}{R_{1}}+\frac{a}{R^{*}} \sqrt{a^{2}-b^{2}}, \\
F_{N}(a) & =\frac{2 E^{*}}{3}\left\{\frac{2 a^{3}}{R_{1}}+\frac{1}{R^{*}}\left(2 a^{2}+b^{2}\right) \sqrt{a^{2}-b^{2}}\right\}, \\
\sigma_{z z}(r ; a) & =-\frac{E^{*}}{\pi}\left\{\begin{array}{l}
\frac{2 \sqrt{a^{2}-r^{2}}}{R_{1}}+\int_{b}^{a} \frac{\left(2 x^{2}-b^{2}\right) \mathrm{d} x}{R^{*} \sqrt{x^{2}-b^{2}} \sqrt{x^{2}-r^{2}}}, \quad r \leq b, \\
\frac{2 \sqrt{a^{2}-r^{2}}}{R_{1}}+\int_{r}^{a} \frac{\left(2 x^{2}-b^{2}\right) \mathrm{d} x}{R^{*} \sqrt{x^{2}-b^{2}} \sqrt{x^{2}-r^{2}}}, \quad b<r \leq a,
\end{array}\right. \\
w(r ; a) & =w_{P}\left(r ; a ; R=R_{1}\right)+w_{P S}\left(r ; a ; R=R^{*}\right), \quad r>a .
\end{aligned}
$$

Here, $w_{P}$ and $w_{P S}$ denote the displacements in the indentation by a complete and cut-off paraboloid:

$$
\begin{gathered}
w_{P}\left(r ; a ; R_{1}\right)=\frac{a^{2}}{\pi R_{1}}\left[\left(2-\frac{r^{2}}{a^{2}}\right) \arcsin \left(\frac{a}{r}\right)+\frac{\sqrt{r^{2}-a^{2}}}{a}\right], \\
w_{P S}\left(r ; a ; R^{*}\right)=\frac{2 a}{\pi R^{*}} \sqrt{a^{2}-b^{2}} \arcsin \left(\frac{a}{r}\right) \\
-\frac{1}{\pi R^{*}}\left[\left(r^{2}-b^{2}\right) \arcsin \left(\frac{\sqrt{a^{2}-b^{2}}}{\sqrt{r^{2}-b^{2}}}\right)\right. \\
\left.-\sqrt{a^{2}-b^{2}} \sqrt{r^{2}-a^{2}}\right] .
\end{gathered}
$$


Fig. 2.35 Stress distribution, normalized to the mean pressure in contact, for indentation by a paraboloid with round cap for $b / a=0.5$ at different values $R_{1} / R^{*}$. The thin solid lines indicate the solutions for the complete paraboloid and the truncated paraboloid

Fig. 2.36 Displacements of the half-space, normalized to the indentation depth, for indentation by a paraboloid with a round cap for $b / a=0.5$ at different values $R_{1} / R^{*}$. The thin solid lines indicate the solutions for the complete paraboloid and the truncated paraboloid
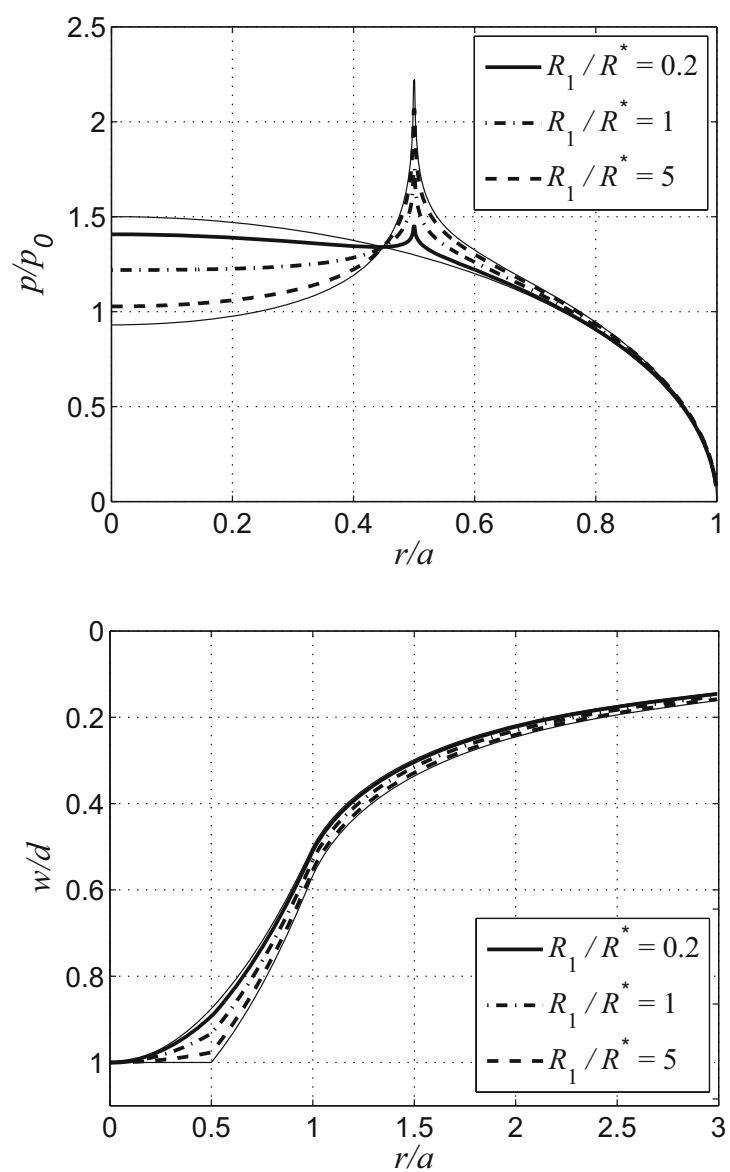

For $R_{1}=R_{2}$, respectively $R^{*} \rightarrow \infty$, we obtain the Hertzian solution from Sect. 2.5.3, and for $R_{1} \rightarrow \infty$ the solution of the truncated paraboloid from Sect. 2.5.10.

Since the solution is determined by two parameters, $b / a$ and $R_{1} / R^{*}$, it would be very ponderous to try to show all solutions for the stresses and displacements in a single diagram. Moreover, the superposition (2.94) already clarifies the structure of the solution. Therefore, the dependencies are shown only for $b / a=0.5$ in Figs. 2.35 and 2.36. One recognizes the limiting cases given by the thin solid lines and the stress singularity at the sharp edge at $r=b$, which is more localized when approaching the parabolic solution. 


\subsubsection{The Cylindrical Flat Punch with a Rounded Edge}

A real flat cylindrical punch will never have a perfectly sharp edge but will always be rounded. The influence of this curvature on the normal contact problem was investigated for both plain and axisymmetric contacts by Schubert (1942) (see also later papers by Ciavarella et al. (1998) and Ciavarella (1999), who presented a solution to the rotationally symmetric problem based on Shtaerman's (1949) general solution). The indenter has the profile (see Fig. 2.37):

$$
f(r)= \begin{cases}0, & r \leq b, \\ \frac{(r-b)^{2}}{2 R}, & r>b,\end{cases}
$$

with the radius of curvature of the rounded corner, $R$, and the radius of the blunt end, $b$. The contact problem is solved according to (2.17) by the relations:

$$
\begin{aligned}
g(x) & = \begin{cases}0, & |x| \\
\frac{R}{R}\left[\sqrt{x^{2}-b^{2}}-b \arccos \left(\frac{b}{|x|}\right)\right], & |x|>b,\end{cases} \\
d(a) & =\frac{a}{R}\left[\sqrt{a^{2}-b^{2}}-b \arccos \left(\frac{b}{a}\right)\right]=\frac{a^{2}}{R}\left(\sin \varphi_{0}-\varphi_{0} \cos \varphi_{0}\right), \\
F_{N}(a) & =\frac{E^{*}}{3 R}\left[\sqrt{a^{2}-b^{2}}\left(4 a^{2}-b^{2}\right)-3 b a^{2} \arccos \left(\frac{b}{a}\right)\right] \\
& =\frac{E^{*} a^{3}}{3 R}\left[\sin \varphi_{0}\left(4-\cos ^{2} \varphi_{0}\right)-3 \varphi_{0} \cos \varphi_{0}\right],
\end{aligned}
$$

with the angle:

$$
\varphi_{0}:=\arccos \left(\frac{b}{a}\right) .
$$

For $b=0$, the Hertzian solution from Sect. 2.5.3 is recovered, and for $R=0$ and $b=a$, i.e., $\varphi_{0}=0$, the solution for the cylindrical flat punch from Sect. 2.5.1.

Fig.2.37 Normal indentation by a flat cylindrical punch with a rounded edge

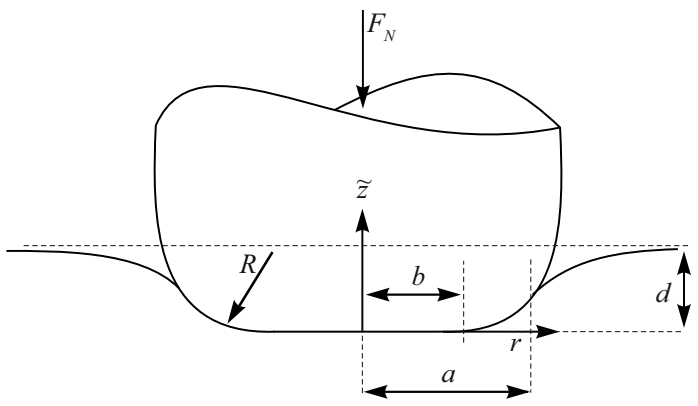


Fig. 2.38 Stress distribution, normalized to the mean pressure in contact, for indentation by a flat punch with rounded corners at different values $b / a$. The thin solid lines indicate the solutions for the paraboloid and the flat punch

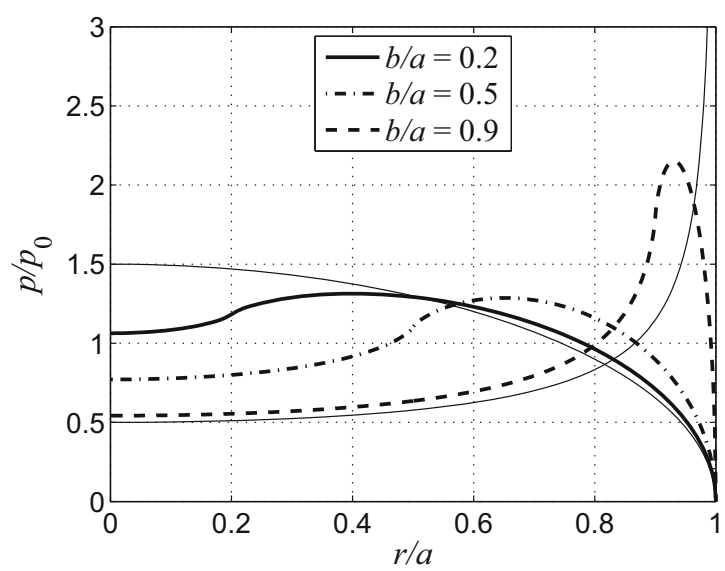

It can be seen from the form of the function $g(x)$ that this contact problem actually represents a sum of two already solved problems and, therefore, the solution can be obtained via an appropriate superposition. It is:

$$
g(x)=g_{P S}(x)-g_{K S}\left(x ; \tan \theta=\frac{b}{R}\right) .
$$

Here $g_{P S}(x)$ denotes the equivalent profile of a truncated paraboloid (see (2.78)) and $g_{K S}$ that of a truncated cone (see (2.72)) whose conical angle of inclination $\theta$ is determined by the relationship $\tan \theta=b / R$. For the stresses and displacements we get:

$$
\begin{aligned}
\sigma_{z z}(r ; a)= & -\frac{E^{*}}{\pi R} \begin{cases}\int_{b}^{a}\left(2 \sqrt{x^{2}-b^{2}}-b \arccos \left(\frac{b}{x}\right)\right) \frac{\mathrm{d} x}{\sqrt{x^{2}-r^{2}}}, & r \leq b, \\
\int_{r}^{a}\left(2 \sqrt{x^{2}-b^{2}}-b \arccos \left(\frac{b}{x}\right)\right) \frac{\mathrm{d} x}{\sqrt{x^{2}-r^{2}}}, & b<r \leq a,\end{cases} \\
w(r ; a)= & \frac{2 d(a)}{\pi} \arcsin \left(\frac{a}{r}\right) \\
& -\frac{2}{\pi}\left[\int_{b}^{a} \frac{x}{R}\left(\sqrt{x^{2}-b^{2}}-b \arccos \left(\frac{b}{x}\right)\right) \frac{\mathrm{d} x}{\sqrt{r^{2}-x^{2}}}\right], \quad r>a .
\end{aligned}
$$

These are shown in Figs. 2.38 and 2.39. Due to the rounded edge, the stress at the edge of the contact - in contrast to the indentation by a flat cylindrical punch-is not singular. 
Fig. 2.39 Displacements, normalized to the indentation depth, for the indentation by a flat punch with rounded corners at different values $b / a$. The thin solid lines indicate the solutions for the paraboloid and the flat punch

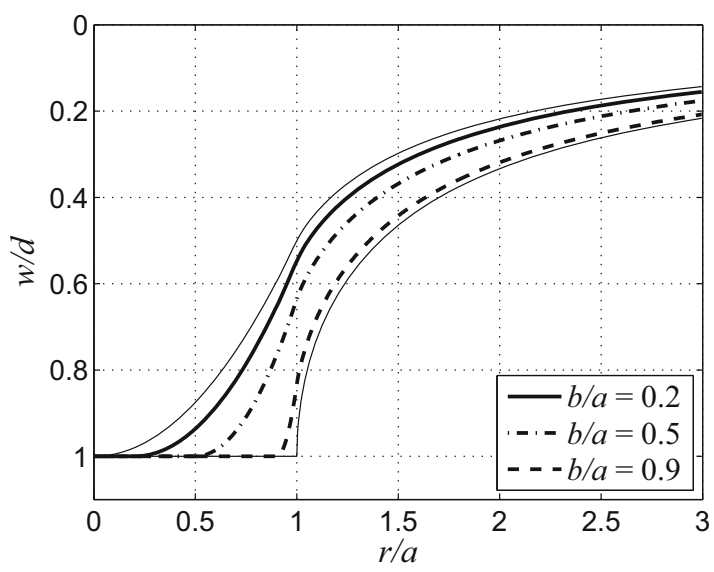

\subsubsection{The Concave Paraboloid (Complete Contact)}

For concave indenters of certain profile geometries, the contact problem can be solved analytically and in closed form using the previously applied methods, assuming the contact area remains compact. Sharp concave corners or edges-such as the cases of a cylinder with a central recess or a concave cone-render this impossible. For these cases of annular contact areas, there sometimes exist semi-analytical solutions in the form of series expansions, which will be detailed in Chap. 10 of this book. The interested reader can also refer to the respective publications of Collins (1963) and Barber (1976, 1983). Complete contact of the concave paraboloid can be ensured if the normal force is large enough. The solution to this problem was discovered by Schubert (1942) (see also Barber 1976). The profile can be characterized by:

$$
f(r)= \begin{cases}-\frac{h r^{2}}{a^{2}}, & r \leq a, \\ \infty, & r>a .\end{cases}
$$

For the notation, see Fig. 2.40.

Fig. 2.40 Normal indentation by a parabolic concave indenter

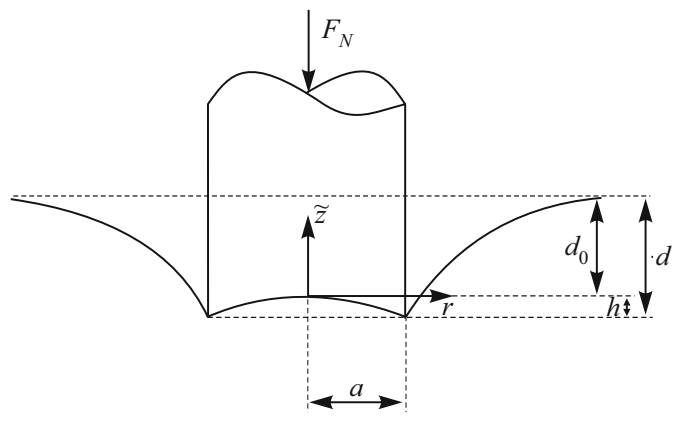


Fig. 2.41 Stress distribution, normalized to the average pressure, for the indentation by concave paraboloid with different values $h / d_{0}$. The thin solid line represents the solution for the flat cylindrical punch

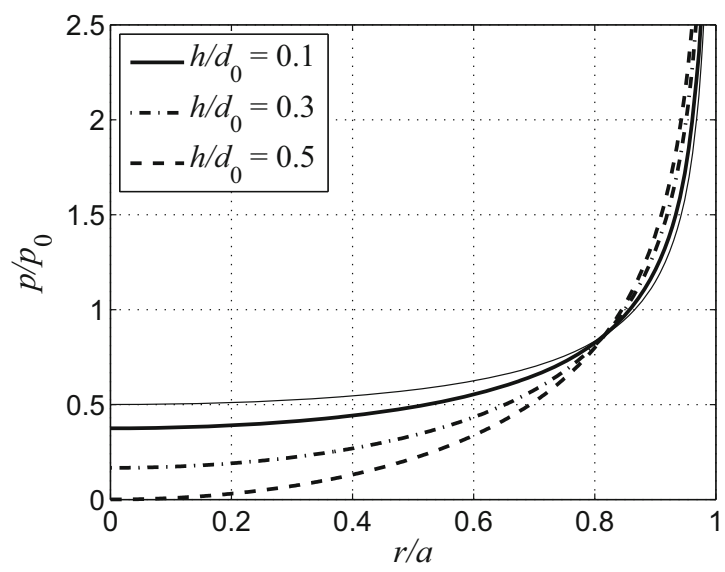

The contact problem is solved according to (2.17) by the relationships:

$$
\begin{aligned}
g(x) & = \begin{cases}-\frac{2 h|x|}{a^{2}} \int_{0}^{|x|} \frac{r \mathrm{~d} r}{\sqrt{x^{2}-r^{2}}}=-\frac{2 h x^{2}}{a^{2}}, & |x| \leq a, \\
\infty, & |x|>a,\end{cases} \\
F_{N}\left(d_{0}\right) & =2 E^{*} a\left(d_{0}+\frac{2 h}{3}\right), \\
\sigma_{z z}\left(r ; d_{0}\right) & =-\frac{E^{*}\left[d_{0} a^{2}-2 h\left(a^{2}-2 r^{2}\right)\right]}{\pi a^{2} \sqrt{a^{2}-r^{2}},} \quad r \leq a, \\
w\left(r ; d_{0}\right) & =\frac{2}{\pi} \int_{0}^{a}\left(d_{0}+\frac{2 h x^{2}}{a^{2}}\right) \frac{\mathrm{d} x}{\sqrt{r^{2}-x^{2}}} \quad \\
& =\frac{2 d_{0}}{\pi} \arcsin \left(\frac{a}{r}\right)+\frac{2 h}{\pi a^{2}}\left(r^{2} \arcsin \left(\frac{a}{r}\right)-a \sqrt{r^{2}-a^{2}}\right), \quad r>a .
\end{aligned}
$$

Setting $h=0$ yields the solution for the flat cylindrical punch. For complete contact, condition $\sigma_{z z}(r=0)<0$ must be satisfied, which leads to the conditions $d_{0}>2 h$, or equivalently, $F_{N}>16 E^{*} a h / 3$. The normalized stresses and displacements are shown in Figs. 2.41 and 2.42.

\subsubsection{The Concave Profile in the Form of a Power-Law (Complete Contact)}

We will briefly focus on the indentation through a concave power profile in the form of:

$$
f(r)=\left\{\begin{array}{ll}
-\frac{h r^{n}}{a^{n}}, & r \leq a, \\
\infty, & r>a
\end{array} \quad n \in \mathbb{R}^{+} .\right.
$$


Fig. 2.42 Displacements of the half-space, normalized to the indentation depth, for the indentation by a concave paraboloid with different values $h / d_{0}$. The thin solid line represents the solution for the flat cylindrical punch

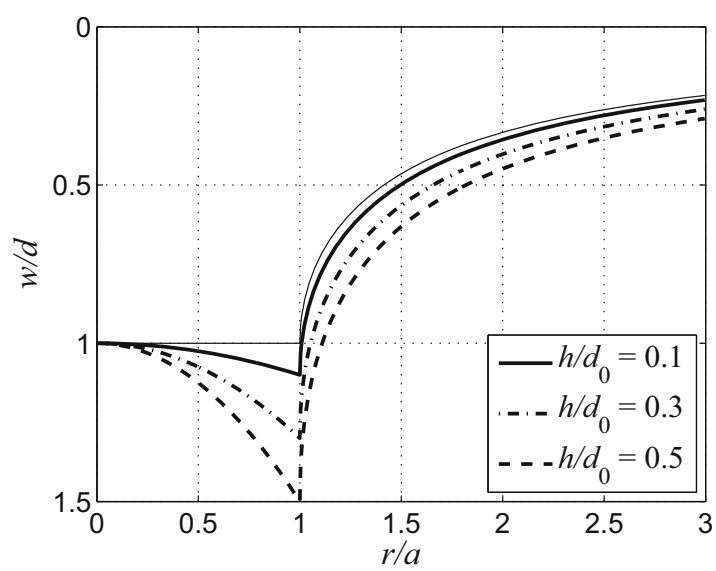

The notations can again be taken from Fig. 2.40. If the normal force is large enough to ensure complete contact, the solution to the contact problem is, following (2.17), given by:

$$
\begin{aligned}
g(x) & = \begin{cases}-\kappa(n) \frac{h|x|^{n}}{a^{n}}, & |x| \leq a \\
\infty, & |x|>a\end{cases} \\
F_{N}\left(d_{0}\right) & =2 E^{*} a\left(d_{0}+\frac{\kappa(n) h}{n+1}\right), \\
\sigma_{z z}\left(r ; d_{0}\right) & =-\frac{E^{*}}{\pi}\left\{\int_{r}^{a}-\frac{\kappa(n) n h x^{n-1}}{a^{n}} \frac{\mathrm{d} x}{\sqrt{x^{2}-r^{2}}}+\frac{d_{0}+\kappa(n) h}{\sqrt{a^{2}-r^{2}}}\right\}, \quad r \leq a \\
w\left(r ; d_{0}\right) & =\frac{2}{\pi} \int_{0}^{a}\left(d_{0}+\frac{\kappa(n) h x^{n}}{a^{n}}\right) \frac{\mathrm{d} x}{\sqrt{r^{2}-x^{2}}}, \quad r>a
\end{aligned}
$$

with the indentation depth in the middle of the contact, $d_{0}$, and the scaling factor

$$
\kappa(n):=\sqrt{\pi} \frac{\Gamma(n / 2+1)}{\Gamma[(n+1) / 2]},
$$

with the gamma function $\Gamma(\cdot)$

$$
\Gamma(z):=\int_{0}^{\infty} t^{z-1} \exp (-t) \mathrm{d} t .
$$

For details relating to integrals occurring in the stresses and displacements, see Sect. 2.5.8. 
One can ask how large the indentation depth must be in order to ensure complete contact. For $n \leq 2$, the minimum of the contact pressure will be in the center of the contact (and the center point will be the last one to make contact during indentation). In this case the condition of complete contact can be determined easily and without evaluating the aforementioned integrals. One obtains that $\sigma_{z z}(r=0)<0$ if

$$
d_{0}>h \frac{\kappa(n)}{n-1}, \quad 1<n \leq 2 .
$$

From this it can be seen that, for a concave cone (and hence $n=1$ ), and in general for all concave profiles with $n \leq 1$, no complete contact can be realized. This was already known to Barber (1976). Therefore, for the relations occurring in (2.105), it must be $n>1$. For exponents $n>2$ the minimum contact pressure in complete contact lies away from the contact midpoint. During indentation, first the contact annulus propagates inside. After a first critical point, contact is established in the contact center and an additional circular contact area grows from the inside. Complete contact is established if the inner contact circle and the outer contact annulus overlap. The determination of the criterion of complete contact for $n>2$ is a nontrivial task (and usually only possible numerically by finding the minimum of the contact pressure and setting the minimal pressure to zero), which was solved by Popov et al. (2018). For a simple example which is possible to analyze in closed form, we can examine the results for the case $n=4$. The pressure distribution for complete contact is given by:

$$
p(r)=-\sigma_{z z}(r ; d)=\frac{E^{*}}{\pi}\left[\frac{3 d+5 h}{3 \sqrt{a^{2}-r^{2}}}-\frac{32 h}{9 a} \sqrt{1-\frac{r^{2}}{a^{2}}}\left(1+\frac{2 r^{2}}{a^{2}}\right)\right] .
$$

Conditions $p^{\prime}\left(r_{c}\right)=0$, and simultaneously $p\left(r_{c}\right)=0$, lead to $3 d=7 h$, i.e., full contact is established for:

$$
d_{0}>\frac{4}{3} h
$$

\subsubsection{The Paraboloid with Small Periodic Roughness (Complete Contact)}

Finally, a simple analytical model of a parabolic indenter with periodic surface roughness is presented. The influence of roughness gains great importance in the treatment of the adhesive normal contact (see Sect. 3.5.14 in Chap. 3). Nonetheless, it can also be of interest for the non-adhesive contact. The contact problem was first solved by Guduru (2007). His solution uses a superposition method, which amounts to the same algorithm as the MDR, i.e., determining the auxiliary function $g(x)$, which basically solves the contact problem.

Let the examined three-dimensional profile have the shape:

$$
f(r)=\frac{r^{2}}{2 R}+h\left(1-\cos \left(\frac{2 \pi}{\lambda} r\right)\right),
$$


with the amplitude $h$ and the wavelength $\lambda$ of the roughness, as well as the usual radius of the paraboloid $R$. Once more we assume a compact contact area. The chapter on adhesive normal contact imposes even stricter limitations requiring $f^{\prime}(r) \geq 0$ (see Sect. 3.5.14). For the equivalent profile we obtain:

$$
\begin{aligned}
g(x) & =|x| \int_{0}^{|x|}\left(\frac{r}{R}+\frac{2 \pi}{\lambda} h \sin \left(\frac{2 \pi}{\lambda} r\right)\right) \frac{\mathrm{d} r}{\sqrt{x^{2}-r^{2}}} \\
& =\frac{x^{2}}{R}+\frac{\pi^{2} h}{\lambda}|x| \mathrm{H}_{0}\left(\frac{2 \pi}{\lambda}|x|\right) .
\end{aligned}
$$

Here, $H_{n}(\cdot)$ denotes the $n$-th order Struve function, which can be expanded as a power series:

$$
\mathrm{H}_{n}(u):=\sum_{k=0}^{\infty} \frac{(-1)^{k}}{\Gamma\left(k+\frac{3}{2}\right) \Gamma\left(k+n+\frac{3}{2}\right)}\left(\frac{u}{2}\right)^{2 k+n+1},
$$

with the Gamma function $\Gamma(\cdot)$ :

$$
\Gamma(z):=\int_{0}^{\infty} t^{z-1} \exp (-t) \mathrm{d} t
$$

From (2.113) the following differentiation property of the Struve function can be proven:

$$
\frac{\mathrm{d}}{\mathrm{d} u}\left[\mathrm{H}_{n}(u)\right]=\mathrm{H}_{n-1}(u)-\frac{n}{u} \mathrm{H}_{n}(u) .
$$

The relationships between the global contact quantities $F_{N}, d$ and $a$ using (2.17) are given by equations:

$$
\begin{aligned}
d(a) & =\frac{a^{2}}{R}+\frac{\pi^{2} h a}{\lambda} \mathrm{H}_{0}\left(\frac{2 \pi}{\lambda} a\right), \\
F_{N}(a) & =\frac{4 E^{*} a^{3}}{3 R}+E^{*} \pi a h\left[\frac{2 \pi}{\lambda} a \mathrm{H}_{0}\left(\frac{2 \pi}{\lambda} a\right)-\mathrm{H}_{1}\left(\frac{2 \pi}{\lambda} a\right)\right] .
\end{aligned}
$$

For the stresses in the contact area we obtain:

$$
\begin{aligned}
& \sigma_{z z}(r ; a)= \\
& -\frac{E^{*}}{\pi}\left\{\frac{2 \sqrt{a^{2}-r^{2}}}{R}+\frac{\pi^{2} h}{\lambda} \int_{r}^{a}\left[\mathrm{H}_{0}\left(\frac{2 \pi}{\lambda} x\right)+\frac{2 \pi}{\lambda} x \mathrm{H}_{-1}\left(\frac{2 \pi}{\lambda} x\right)\right] \frac{\mathrm{d} x}{\sqrt{x^{2}-r^{2}}}\right\}, \\
& \quad r \leq a
\end{aligned}
$$


Fig. 2.43 Pressure distribution, normalized to the average pressure in the contact, for the indentation by a paraboloid with small periodic roughness at $h R / \lambda^{2}=0.1$ and different values $\lambda / a$. The thin solid line represents the solution for the smooth paraboloid

Fig. 2.44 Profile cross-section of the threedimensional rotationally symmetric profile, and the equivalent profile within the framework of the MDR for a paraboloid with small periodic roughness
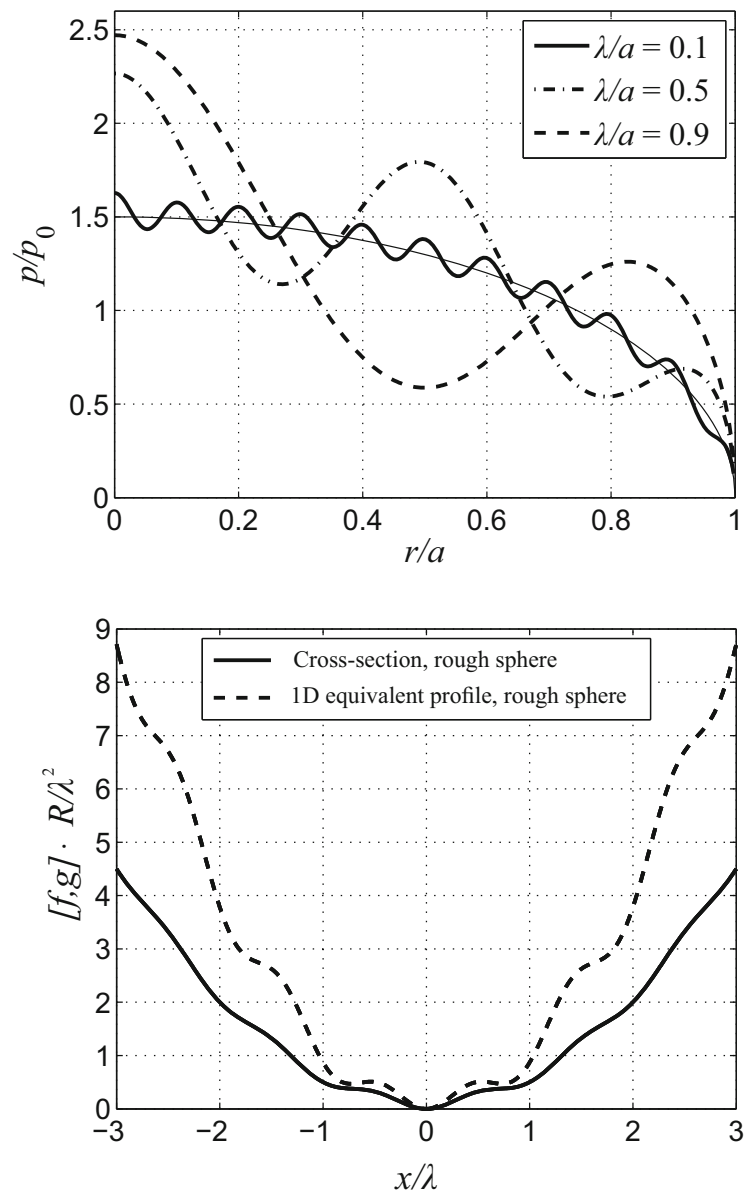

and the displacements outside the contact area are given by:

$$
\begin{aligned}
w(r ; a)= & \frac{2 d(a)}{\pi} \arcsin \left(\frac{a}{r}\right)-\frac{1}{\pi R}\left(r^{2} \arcsin \left(\frac{a}{r}\right)-a \sqrt{r^{2}-a^{2}}\right) \\
& -\frac{2 \pi}{\lambda} h \int_{0}^{a} x \mathrm{H}_{0}\left(\frac{2 \pi}{\lambda} x\right) \frac{\mathrm{d} x}{\sqrt{r^{2}-x^{2}}}, \quad r>a .
\end{aligned}
$$

The stresses normalized to the average pressure in the contact are displayed in Fig. 2.43. The influence of the periodic roughness on the pressure distribution is quite apparent. Figure 2.44 shows the three-dimensional profile and also the equivalent one-dimensional profile. 


\subsubsection{Displacement in the Center of an Arbitrary Axially Symmetric Pressure Distribution}

The displacement $w_{1 D}(x)$ of the one-dimensional MDR-model is given by (2.16):

$$
w_{1 D}(x)=\frac{q_{z}(x)}{E^{*}}=\frac{2}{E^{*}} \int_{x}^{\infty} \frac{r p(r)}{\sqrt{r^{2}-x^{2}}} \mathrm{~d} r .
$$

Setting $x=0$ yields the indentation depth $d_{0}$, which in the context of the MDR is defined as the vertical displacement of the coordinate origin:

$$
d_{0}=w_{1 D}(x=0)=w(r=0)=\frac{2}{E^{*}} \int_{0}^{\infty} p(r) \mathrm{d} r .
$$

\subsubsection{Contacts with Sharp-Edged Elastic Indenters}

Flat cylindrical indenters, as well as all other indenters which generate singularities in the stress distribution (conical indenter and truncated cone, etc.), violate the necessary conditions of the half-space approximation at least for one of the contacting bodies. While the given solutions are accurate for rigid profiles, they must be modified for elastic profiles. The relationship of the elastic moduli of the indenter and the half-space, as well as the angle at the sharp edge of the indenter, determine whether the stress concentration at the sharp edge result in different singularities or even no singularity at all.

This problem was systematically examined by Rao (1971). Rao considered the stress and displacement fields in the vicinity of the sharp edge for widely varying classes of problems. Here, we will limit our consideration to the frictionless normal contact between an axisymmetric elastic indenter and an elastic half-space. The used notation is displayed in Fig. 2.45.

The stress state in the region of the sharp edge is approximately two-dimensional, and the normal stress at distance $s$ from the sharp edge is given by:

$$
\sigma_{z z} \sim s^{\lambda-1}
$$

Fig. 2.45 Edge of an elastic indenter (diagram visualizing the notations used)

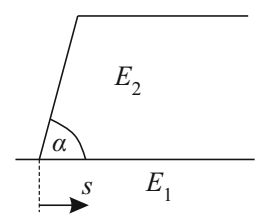


where $\lambda$ indicates the smallest eigenvalue of the respective boundary value problem. The eigenvalue equation of the considered problem can be written as:

$$
\begin{aligned}
& \tan (\pi \lambda)[\lambda \sin (2 \alpha)+\sin (2 \lambda \alpha)] \\
& +e\left[1-\cos (2 \lambda \alpha)-\lambda^{2}(1-\cos (2 \alpha))\right]=0 .
\end{aligned}
$$

Here, $\alpha$ is the angle at the sharp edge (measurement taken within the indenting body) and $e=E_{2} / E_{1}$ is the relationship of the elasticity moduli of the indenter (index "2") and the half-space (index "1"). Interestingly, the respective Poisson's ratios have no effect on this characteristic equation.

A singularity in the stress distribution occurs at the sharp edge if the smallest non-trivial solution (2.122) is smaller than one. The limiting case, $\lambda=1$, arises precisely when the relationship of the moduli is set to:

$$
e_{\text {crit }}=\frac{\pi \cos \alpha}{\sin \alpha-\cos \alpha} .
$$

Greater values of $e$ will result in singularities. However, (2.123) only has a positive solution for $e$ if $\alpha \leq \pi / 2$. Therefore, in principle, greater angles always result in singularities, whose concrete shape is determined by the solution of the eigenvalue equation (2.122). As an example, for the rigid flat cylindrical punch with $\alpha=\pi / 2$ and $e \rightarrow \infty$, the result is the familiar singularity of the Boussinesq solution, $\lambda=1 / 2$.

How can the stresses be expressed in the entire contact area? For this, Jordan and Urban (1999) proposed the following expression for rectangular indenters in a plane (which can be directly applied to rotationally symmetric problems with cylindrical flat punches):

$$
\sigma_{z z}(r ; a, \lambda)=-F_{N} \cdot M(\lambda, a)\left(a^{2}-r^{2}\right)^{\lambda-1} .
$$

The case of the rigid cylindrical punch coincides with the known Boussinesq solution, and the stresses exhibit the required singularity property (2.121) since for $s=a-r \ll a$ :

$$
a^{2}-r^{2}=(a-r)(a+r)=s(a+r) \approx 2 a s .
$$

The function $M(\lambda, a)$ is derived from the normalization:

$$
F_{N}=-2 \pi \int_{0}^{a} \sigma_{z z}(r) r \mathrm{~d} r,
$$

and therefore:

$$
M(\lambda, a)=\frac{\lambda}{\pi a^{2 \lambda}} .
$$




\subsection{Mossakovskii Problems (No-Slip)}

In the preceding sections, we considered frictionless normal contact problems. Assuming different elastic materials, the material points of the contacting surfaces will experience differing radial displacements. If we set aside the unrealistic assumption of ideally frictionless surfaces $(\mu=0)$, this slip will always cause radial tangential stresses. Consequently, the frictionless normal contact can only be considered the theoretical limiting case. The contact area generally consists of an inner stick zone and an outer slip zone. This normal contact problem with friction was examined by Spence (1975). It is supremely complicated, and thus only permits numerical solutions. Instead, we will just consider the other theoretical limiting case which describes complete stick within the contact area $(\mu \rightarrow \infty)$. Figure 2.46 illustrates the indentation of an elastic half-space by a rigid curved indenter with complete stick. Also displayed are the displacement paths of individual surface points. Surface points that contact the indenter during the indentation process stick completely, eliminating the possibility of any further radial displacement. This condition is expressed through the boundary condition:

$$
\frac{\partial u_{r}(r, a)}{\partial a}=0, \quad r \leq a,
$$

and it is used instead of the boundary condition for the frictionless normal contact according to (2.4) which assumes a frictionless contact. Here, it should be noted that for the contact of two elastic bodies, the radial displacement from (2.128) simply represents the relative radial displacement of the surface points.

The solution of the normal contact problem with complete stick for arbitrary axially symmetric normal contacts goes back to Mossakovskii (1963). In 1954, he had already developed the solution for the contact of the flat cylindrical punch. Therefore, normal contacts with complete stick are also referred to as Mossakovskii problems. Their solutions are significantly more complex than frictionless contacts, and the simplest approach to solving them is with the MDR. This requires redefining the spring stiffness compared to (2.5) and deriving the equivalent one-dimensional

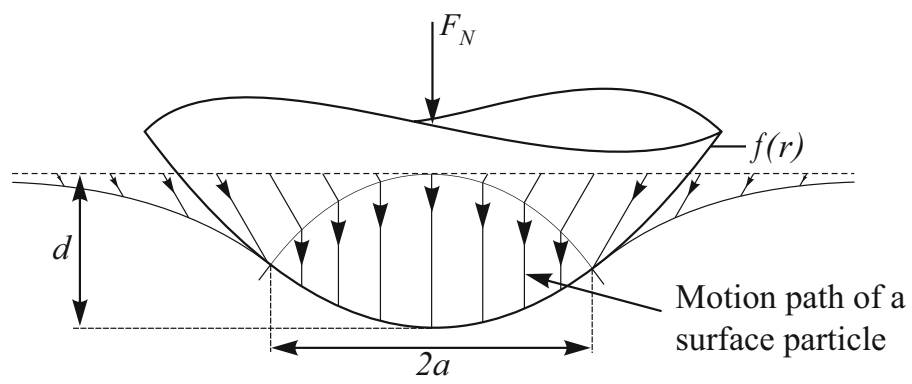

Fig. 2.46 Illustrating the boundary condition of full stick for the indentation of an elastic halfspace by a rigid indenter (modeled after Spence 1968) 
profile from an equation significantly more complicated than (2.6). The calculation of the global relationships between normal force, indentation depth, and contact radius follows the exact same template as described in Sect. 2.3.2. To describe the normal contact between two completely sticking elastic bodies, the spring stiffness is set to:

$$
\Delta k_{z}=\frac{E^{*}}{2 \beta_{D}} \ln \left(\frac{1+\beta_{D}}{1-\beta_{D}}\right) \Delta x .
$$

Here, $E^{*}$ denotes the effective elasticity modulus defined in (2.1):

$$
\frac{1}{E^{*}}=\frac{1-v_{1}^{2}}{E_{1}}+\frac{1-v_{2}^{2}}{E_{2}},
$$

and $\beta_{D}$ Dundur's second constant:

$$
\beta_{D}:=\frac{\left(1-2 v_{1}\right) G_{2}-\left(1-2 v_{2}\right) G_{1}}{2\left(1-v_{1}\right) G_{2}+2\left(1-v_{2}\right) G_{1}} .
$$

Many contact problems with complete stick featured in the literature make the simplifying assumption of one body being rigid. In this case, the spring stiffness from (2.129) is simplified to:

$$
\Delta k_{z}=\frac{2 G \ln (3-4 v)}{1-2 v} \Delta x .
$$

The relationship between the three-dimensional profile $f(r)$ and the equivalent onedimensional profile $g(x)$ is given by equation:

$$
\begin{aligned}
& f(r)=\frac{2}{\pi} \int_{0}^{r} \frac{1}{\sqrt{r^{2}-x^{2}}} \int_{0}^{x} g^{\prime}(t) \cos \left(\vartheta \ln \left(\frac{x-t}{x+t}\right)\right) \mathrm{d} t \mathrm{~d} x \\
& \text { with } \vartheta:=\frac{1}{2 \pi} \ln \left(\frac{1+\beta_{D}}{1-\beta_{D}}\right) .
\end{aligned}
$$

For $\beta_{D}=0$, it coincides with the inverse transform of the profile for a frictionless contact according to (2.7). While explicitly solving for $g(x)$ is possible in principle, it leads to either a very unwieldy, extremely complicated calculation which can be referenced in Fabrikant's (1986) work, or a notation using the Mellin inverse transform (Spence 1968). We will forgo providing an explicit expression since an analytical calculation of the equivalent one-dimensional profile $g(x)$ for a given axially symmetric profile $f(r)$ is generally only possible using numerical methods anyway. The sole exception seems to be the power-law profile, which we will examine in greater detail in Sect. 2.6.2. To calculate the respective one-dimensional equivalent profile, the implicit formulation in (2.133) will prove sufficient.

The elasticity parameter $\vartheta$ in (2.133) illustrates a major difference to the frictionless contact: the equivalent one-dimensional profile is no longer exclusively 
dependent on the geometry of the axially symmetric contact; it is also affected by the elastic properties of the contacting bodies.

As previously mentioned, the calculation of the relationships between indentation depth, contact radius, and normal force follows the same template as the frictionless normal contact. Only the modified spring stiffness from (2.129) and the equivalent profile determined by (2.133) must be accounted for. We will summarize the essential equations. First, the surface displacement of the Winkler foundation is determined to be:

$$
w_{1 D}(x)=d-g(x) .
$$

At the edge of all non-adhesive contacts, the displacement must be zero, thus formulating a condition for calculating the indentation depth:

$$
w_{1 D}( \pm a)=0 \quad \Rightarrow \quad d=g(a)
$$

Additionally, the sum of all spring forces must balance out the applied normal force. Consideration of the modified stiffness in accordance with (2.129) yields:

$$
F_{N}=\frac{E^{*}}{\beta_{D}} \ln \left(\frac{1+\beta_{D}}{1-\beta_{D}}\right) \int_{0}^{a} w_{1 D}(x) \mathrm{d} x .
$$

Naturally, the local quantities obey different calculation formulas compared to the frictionless contact. Apart from the normal surface displacement and the pressure distribution, the tangential stresses within the contact area are also of importance. Said quantities can be determined solely from the known normal displacement of the Winkler foundation. Here, we will state them for the special case of a rigid indenter pressed into an elastic half-space:

$$
\begin{aligned}
p(r, a) & =-\frac{8 G(1-v) \ln (3-4 v)}{\pi^{2}(1-2 v) \sqrt{3-4 v}} \int_{r}^{a} x w_{1 d}^{\prime}(x) \int_{0}^{r} \frac{\cos \left[\vartheta \ln \left(\frac{x+t}{x-t}\right)\right]}{\sqrt{r^{2}-t^{2}}\left(x^{2}-t^{2}\right)} \mathrm{d} t \mathrm{~d} x \\
\tau_{z r}(r, a) & =\frac{8 G(1-v) \ln (3-4 v)}{\pi^{2}(1-2 v) \sqrt{3-4 v}} \int_{r}^{a} \frac{x}{r} w_{1 d}^{\prime}(x) \int_{0}^{r} \frac{\sin \left[\vartheta \ln \left(\frac{x+t}{x-t}\right)\right] t}{\sqrt{r^{2}-t^{2}}\left(x^{2}-t^{2}\right)} \mathrm{d} t \mathrm{~d} x \\
w(r, a) & =-\frac{4(1-v)}{\pi \sqrt{3-4 v}} \int_{0}^{a} w_{1 d}^{\prime}(x) \int_{0}^{x} \frac{\cos \left[\vartheta \ln \left(\frac{x+t}{x-t}\right)\right]}{\sqrt{r^{2}-t^{2}}} \mathrm{~d} t \mathrm{~d} x .
\end{aligned}
$$




\subsubsection{The Cylindrical Flat Punch}

The normal contact of a rigid flat cylindrical punch with an elastic half-space with complete stick was initially solved by Mossakovskii (1954). Within the framework of the MDR, the equivalent plane profile is derived from the cross-section of the flat indenter in the $x-z$-plane-no change in the geometry is required. The displacement of the Winkler foundation is then given by:

$$
w_{1 D}(x)=d[\mathrm{H}(x+a)-\mathrm{H}(x-a)],
$$

where $\mathrm{H}(\cdot)$ represents the Heaviside step function. Substituting this into (2.136) under consideration of the rigidity of the indenter yields the normal force:

$$
F_{N}=\frac{4 G \ln (3-4 v)}{1-2 v} d a .
$$

The contact stiffness then takes on the form:

$$
k_{z}^{\mathrm{M}}:=\frac{\mathrm{d} F_{N}}{\mathrm{~d} d}=\frac{4 G \ln (3-4 v)}{1-2 v} a .
$$

The validity of this contact stiffness for arbitrary axially symmetric contacts follows immediately from Mossakovskii's (1963) work. Accordingly, the incremental difference of two-contact configurations with the contact radii $a$ and $a+\mathrm{d} a$ is equivalent to the infinitesimal indentation of an elastic half-space by a cylindrical flat punch with radius $a$. This applies regardless of whether a normal contact with complete stick or a frictionless contact is being considered. Nevertheless, the works of Borodich and Keer (2004) and also Pharr et al. (1992) are still frequently cited, which prove the universal validity of the normal contact stiffness using a different, more complex approach. For the frictionless normal contact, the contact stiffness is given by (2.21). A comparison of the two values of contact stiffness reveals that the contact stiffness for complete stick is generally greater than for frictionless contact. This is a direct result of the suppressed relative displacement of the contacting surfaces. The contact stiffness only coincides for incompressible materials since, in this case, no tangential forces arise in the contact area due to the radial displacement of the material being restricted. For the ratio between the contact stiffness for complete stick and frictionless contact, it follows that:

$$
\frac{k_{z}^{\mathrm{M}}}{k_{z}}=\frac{(1-v) \ln (3-4 v)}{1-2 v} .
$$

For common materials with Poisson's ratios in the range of $0 \leq v \leq 0.5$, the contact stiffness for complete stick is at most $10 \%$ greater than for frictionless normal contacts. This maximum is reached at $v=0$. For synthetic materials characterized by negative Poisson's ratios, the relative discrepancy can reach values of up to $30 \%$, which is documented in Fig. 2.47. Viewed over the entire physical domain, the relative discrepancy decreases monotonically with a rising Poisson's ratio. The relative discrepancy reaches its maximum for the limiting case $v \rightarrow-1$. It should be noted that both values of contact stiffness approach infinity for $v \rightarrow-1$. At this point, the 


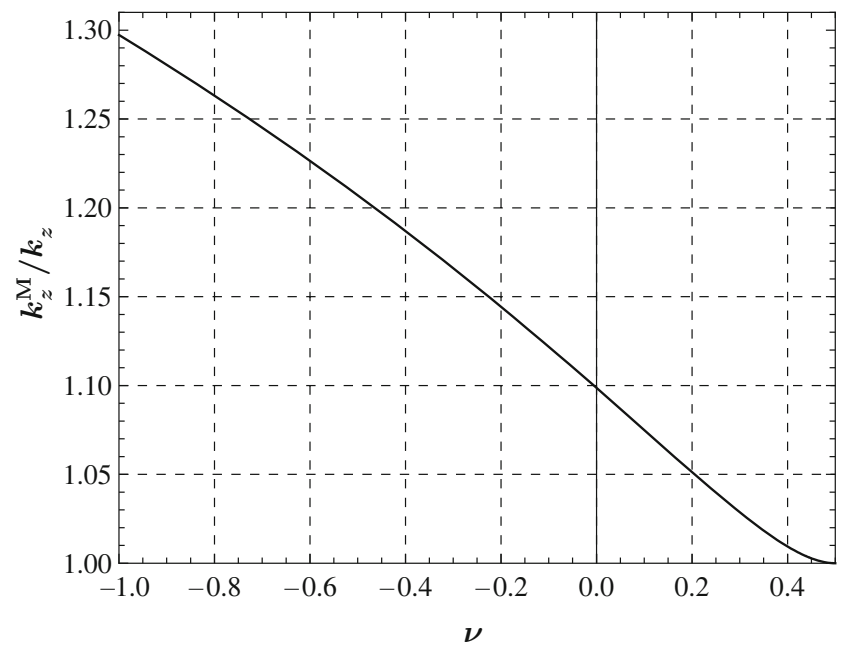

Fig. 2.47 Relationship between the normal contact stiffness for complete stick and normal contact stiffness for frictionless contacts as a function of the Poisson's ratio $v$

contact compliance $\Pi$, which represents the inverse of the stiffness, is zero. Argatov et al. (2012) examined the impact of negative Poisson's ratios on on the stress distribution, thereby discovering that the location of the greatest shear stress moves towards the surface for smaller Poisson's ratios. This is because of the increasing tangential stresses in the contact area for decreasing Poisson's ratios. Figure 2.48 puts in contrast the differing contact compliances for Boussinesq and Mossakovskii

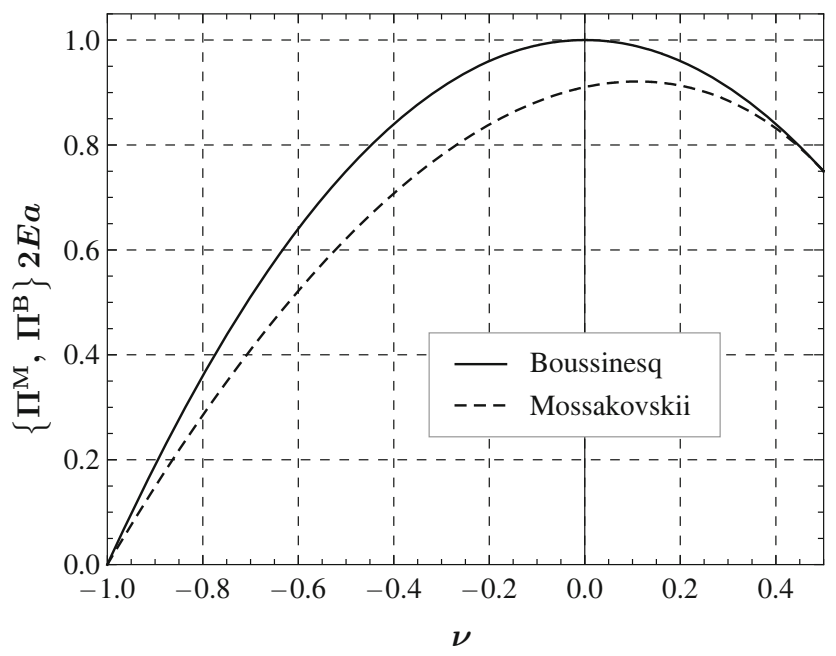

Fig. 2.48 Comparison of the normalized normal contact compliances as functions of the Poisson's ratio $v$ for Boussinesq and Mossakovkii problems 


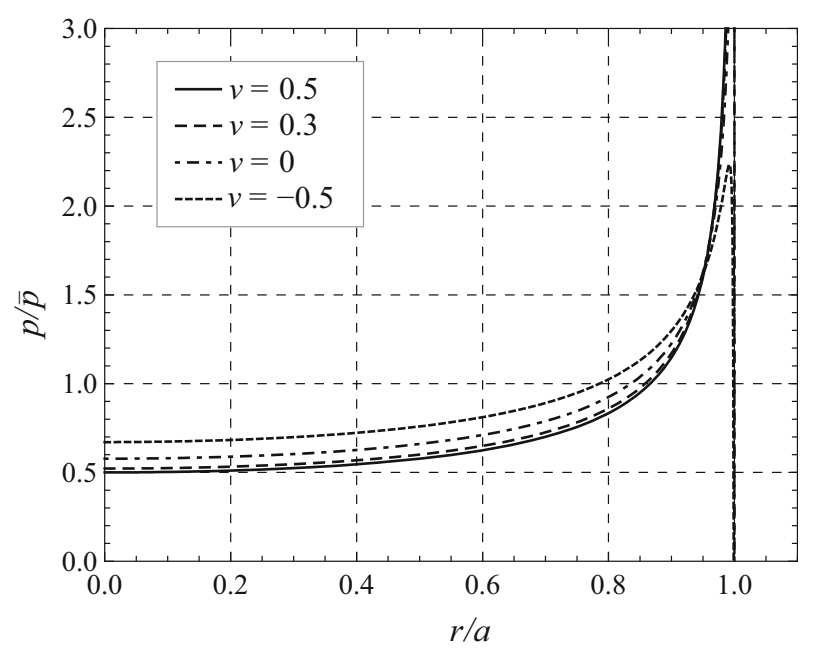

Fig. 2.49 Pressure distribution for the indentation by a flat cylindrical indenter for different Poisson's ratios $v$, normalized to the average pressure $\bar{p}$

problems. It is apparent that the compliance maximum of Mossakovskii problems are not located at $v=0$ but instead at $v \approx 0.11$, as stated by Argatov et al. (2012).

The derivative of the profile (2.138) with respect to the coordinate $x$ is required for calculating the local quantities according to (2.137), and is:

$$
w_{1 D}^{\prime}(x)=d[\delta(x+a)-\delta(x-a)],
$$

with the Delta function $\delta(\cdot)$. Taking into account its filtering properties, it follows from (2.137) that the solutions for the local quantities are:

$$
\begin{aligned}
p(r, a) & =\frac{8 G a d(1-v) \ln (3-4 v)}{\pi^{2}(1-2 v) \sqrt{3-4 v}} \int_{0}^{r} \frac{\cos \left[\vartheta \ln \left(\frac{a+t}{a-t}\right)\right]}{\sqrt{r^{2}-t^{2}}\left(a^{2}-t^{2}\right)} \mathrm{d} t \\
\tau_{z r}(r, a) & =-\frac{8 G a d(1-v) \ln (3-4 v)}{\pi^{2}(1-2 v) \sqrt{3-4 v}} \frac{1}{r} \int_{0}^{r} \frac{\sin \left[\vartheta \ln \left(\frac{a+t}{a-t}\right)\right] t}{\sqrt{r^{2}-t^{2}}\left(a^{2}-t^{2}\right)} \mathrm{d} t \\
w(r, a) & =\frac{4 d(1-v)}{\pi \sqrt{3-4 v}} \int_{0}^{a} \frac{\cos \left[\vartheta \ln \left(\frac{a+t}{a-t}\right)\right]}{\sqrt{r^{2}-t^{2}}} \mathrm{~d} t .
\end{aligned}
$$

They correspond exactly to the quantities calculated by Mossakovskii (1963) and Spence (1968). Regrettably, the remaining integrals are only solvable using numerical methods. The pressure distribution normalized to the average pressure $\bar{p}$ within the contact area is visualized for different Poisson's ratios in Fig. 2.49. For incompressible materials, i.e., $v=1 / 2$, the curve exactly matches the one for the 


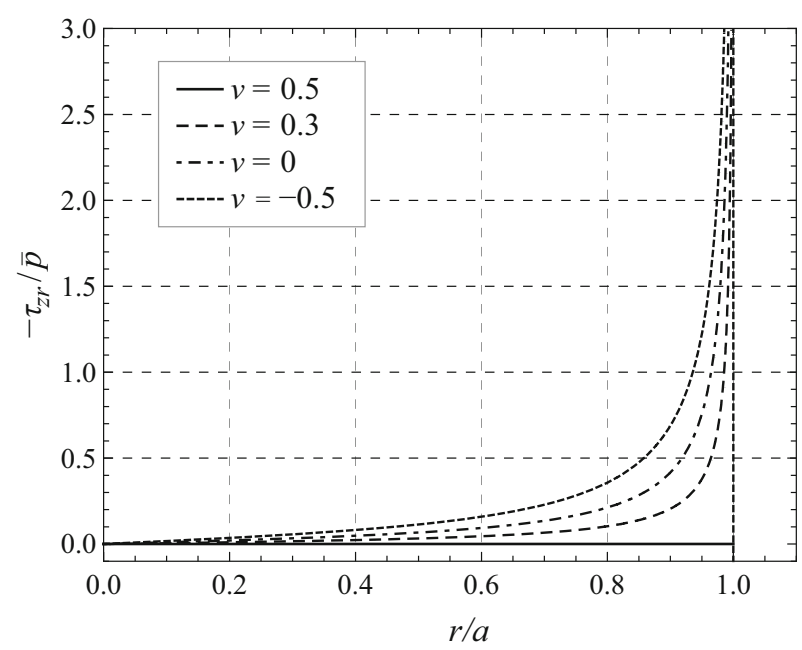

Fig. 2.50 Tangential stresses for the indentation by a flat cylindrical indenter for different Poisson's ratios $v$, normalized to the average pressure $\bar{p}$

frictionless contact (compare this to Fig. 2.5). For regular positive Poisson's ratios, there is no distinguishable difference between the graphs. Only once the Poisson's ratios drop to negative values the pressure in the center notably increases. There is characteristic behavior of the stresses solely at the edge of the contact, which is already visible in solution (2.143). Here, stress oscillations occur, even leading to tensile stresses. On the one hand, these fluctuations can be viewed as indicators that the assumption of complete stick is self-contradictory for normal contact, indicating that slip at the contact edge is unavoidable (Zhupanska 2009). On the other hand, the oscillations are localized so close to the contact edge that this zone need not be ascribed significant importance. ${ }^{1}$

The tangential stresses in Fig. 2.50 are zero at the center of the contact and feature a singularity at the edge that is comparable to the one caused by the normal stresses. For the incompressible case, no tangential stresses occur in the contact area since the material resists radial displacements. Expanding the examination to the contact between two elastic materials, the tangential stresses in the contact area are zero when Dundur's second constant, defined in (2.131), vanishes. This condition is, indeed, satisfied for the contact between a rigid and an incompressible medium.

The curve of the normal surface displacement normalized to the respective indentation depth is shown in Fig. 2.51. The curves for $v=1 / 2$ and $v=0.3$ are nearly indistinguishable, so that their shape can be approximated by the displacements for a frictionless normal contact described by (2.22). A more detailed analysis of the displacements can be referenced in the publication of Fabrikant (1986).

\footnotetext{
${ }^{1}$ In the plane case, the normal stresses initially changes signs at $x=0.9997 a$.
} 


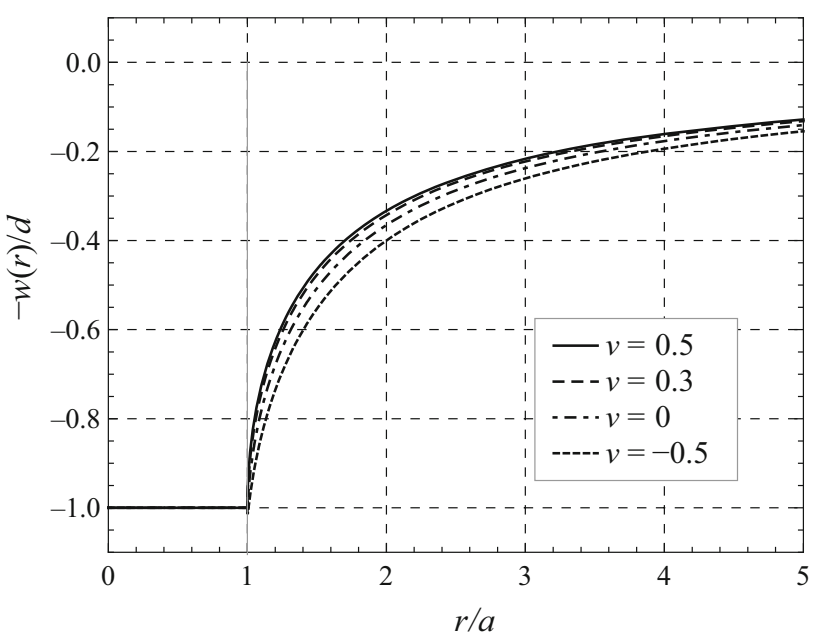

Fig. 2.51 Normal surface displacement of the half-space for indentation by a flat cylindrical indenter for different Poisson's ratios $v$, normalized to the indentation depth $d$

\subsubsection{The Profile in the Form of a Power-Law}

The normal contact of a profile in the shape of a power-law was examined for the case of complete stick by both Mossakovskii (1963) and Spence (1968). As shown for the frictionless contact, a power-law profile is mapped to a power-law profile:

$$
f(r)=c_{n} r^{n} \quad \mapsto \quad g(x)=\kappa(n, v) f(|x|) \quad \text { for } n \in \mathbb{R}^{+} .
$$

The scaling factor $\kappa(n, v)$ can be determined by simply substituting (2.144) in (2.133). A complicated integration is unnecessary; a trivial normalization of the integral variables is sufficient. This leads to:

$$
\begin{aligned}
\kappa(n, v) & =\frac{\sqrt{\pi} \Gamma\left(1+\frac{n}{2}\right)}{\Gamma\left(\frac{1+n}{2}\right) n I^{*}(n)} \text { with } \\
I^{*}(n) & :=\int_{0}^{1} t^{n-1} \cos \left(\vartheta \ln \left(\frac{1-t}{1+t}\right)\right) \mathrm{d} t,
\end{aligned}
$$

with the definition of $\vartheta$ from (2.133). A comparison of the scaling factors to the ones for the frictionless contact is easily performed by setting $\beta_{D}=0$, i.e., by assuming similar elastic materials and yielding $\vartheta=0$, and consequently, $n I^{*}(n)=1$. From (2.145), we obtain the scaling factors for the frictionless contact defined in (2.61). In the following, we will operate under the assumption of one rigid body. In this case, the other body must be incompressible to prevent tangential stresses from occurring in the contact surface. The limiting curve for 


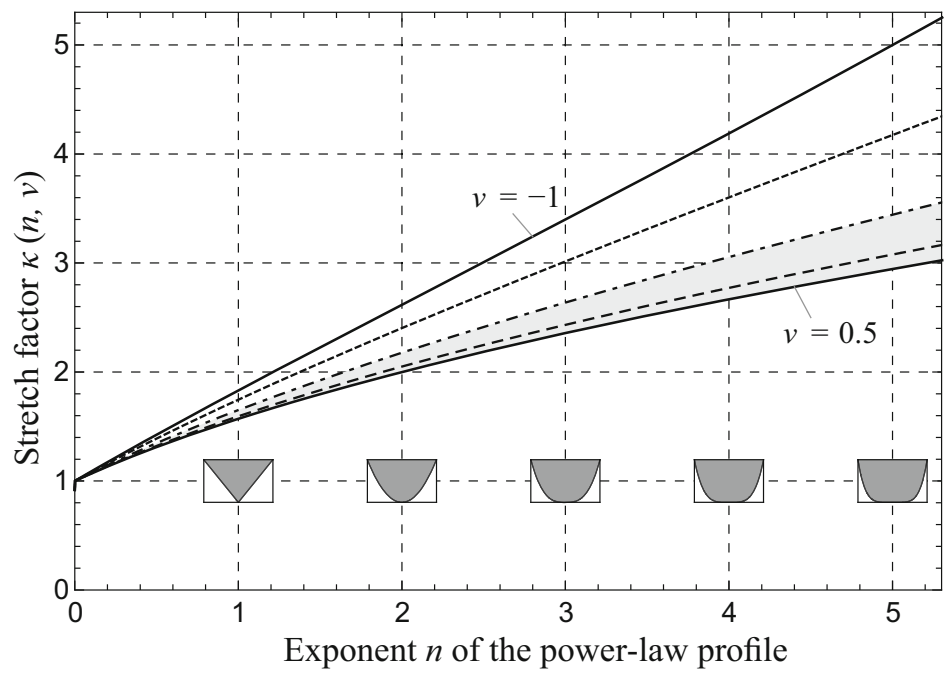

Fig. 2.52 Scaling factors $\kappa$ as a function of the exponent $n$ of the power-law profile for different Poisson's ratios $v$; the area shaded in gray represents common materials $(0 \leq v \leq 0.5)$

$v=1 / 2$ in Fig. 2.52 must exactly coincide with the curve from Fig. 2.21, which displays the scaling factor as a function of the exponents of the profile function for the frictionless contact.

In contrast, complete stick in the normal contact between a rigid power-law indenter and a compressible, elastic half-space results in tangential stresses in the contact surface. The full description of such a contact requires greater scaling factors, also given in Fig. 2.52. The smaller the Poisson's ratio, the greater the scaling factor. The area shaded in gray represents the range of values of the scaling factors for common materials.

Since the integral $I^{*}(n)$ in formula (2.145) of the scaling factor is generally only solvable numerically, the corresponding value for selected Poisson's ratios and exponents is provided in Table 2.2. These are of particular importance for axially symmetric profiles that are either defined by a polynomial or a Taylor expansion.

The equivalent plane profile was already determined in (2.144). Applying formulas (2.135) and (2.136) yields the indentation depth and the normal force as a function of the contact radius:

$$
\begin{aligned}
d(a) & =\kappa(n, v) c_{n} a^{n}, \\
F_{N}(a) & =\frac{4 G \ln (3-4 v)}{1-2 v} \frac{n}{n+1} \kappa(n, v) c_{n} a^{n+1} .
\end{aligned}
$$

The calculation and graphical representation of surface stresses and displacements will be omitted at this point. However, we will analyze the particular cases of the conical $(n=1)$ and parabolic $(n=2)$ contact. 
Table 2.2 Stretch factor $\kappa$ as a function of the exponent of the power-law and Poisson's ratio

\begin{tabular}{|c|c|c|c|c|c|c|}
\hline & \multicolumn{5}{|c|}{ Poisson's ratio $v$} \\
\hline & & -1 & -0.5 & 0 & 0.3 & 0.5 \\
\hline \multirow{11}{*}{$\begin{array}{l}\text { Exponent } n \\
\text { of the } \\
\text { power-law } \\
\text { profile }\end{array}$} & 0.5 & 1.429 & 1.389 & 1.348 & 1.322 & 1.311 \\
\hline & 1 & 1.831 & 1.746 & 1.651 & 1.594 & 1.571 \\
\hline & 2 & 2.617 & 2.402 & 2.177 & 2.049 & 2 \\
\hline & 3 & 3.398 & 3.014 & 2.638 & 2.433 & 2.356 \\
\hline & 4 & 4.189 & 3.602 & 3.056 & 2.771 & 2.667 \\
\hline & 5 & 5.000 & 4.175 & 3.444 & 3.077 & 2.945 \\
\hline & 6 & 5.840 & 4.739 & 3.811 & 3.360 & 3.2 \\
\hline & 7 & 6.714 & 5.298 & 4.159 & 3.623 & 3.436 \\
\hline & 8 & 7.626 & 5.855 & 4.494 & 3.872 & 3.657 \\
\hline & 9 & 8.582 & 6.412 & 4.816 & 4.107 & 3.866 \\
\hline & 10 & 9.588 & 6.971 & 5.123 & 4.332 & 4.063 \\
\hline
\end{tabular}

\subsubsection{The Cone}

We will now consider the normal contact of a rigid cone and a planar elastic halfspace under the condition of complete stick. The shape of the profile function is given by:

$$
f(r)=r \tan \theta
$$

(see Fig. 2.7). The equivalent plane profile follows as a special case of (2.144), where the scaling factor (2.145) must be determined. An analytical expression for the integral $I^{*}$, and consequently the stretch factor (solely in this case), is published in literature (see Spence 1968):

$$
\kappa(1, v)=\frac{\pi}{2 I^{*}(1)}=\frac{\pi}{2} \frac{1-2 v}{\pi \vartheta \sqrt{3-4 v}}=\frac{\pi(1-2 v)}{\ln (3-4 v) \sqrt{3-4 v}} .
$$

The equivalent profile is then:

$$
g(x)=\frac{\pi(1-2 v)}{\ln (3-4 v) \sqrt{3-4 v}}|x| \tan \theta .
$$

Substituting this last result into formulas (2.135) and (2.136), followed by a basic calculation, leads to the indentation depth and normal force

$$
\begin{aligned}
d(a) & =\frac{\pi(1-2 v)}{\ln (3-4 v) \sqrt{3-4 v}} a \tan \theta, \\
F_{N}(a) & =\frac{2 G \pi}{\sqrt{3-4 v}} a^{2} \tan \theta .
\end{aligned}
$$

We omit explicitly providing the stresses and normal surface displacements since, once again, the integrals in expression (2.137) can only be solved numerically. 
Fig. 2.53 Pressure distribution in the conical contact for different Poisson's ratios $v$, normalized to the average pressure $\bar{p}$

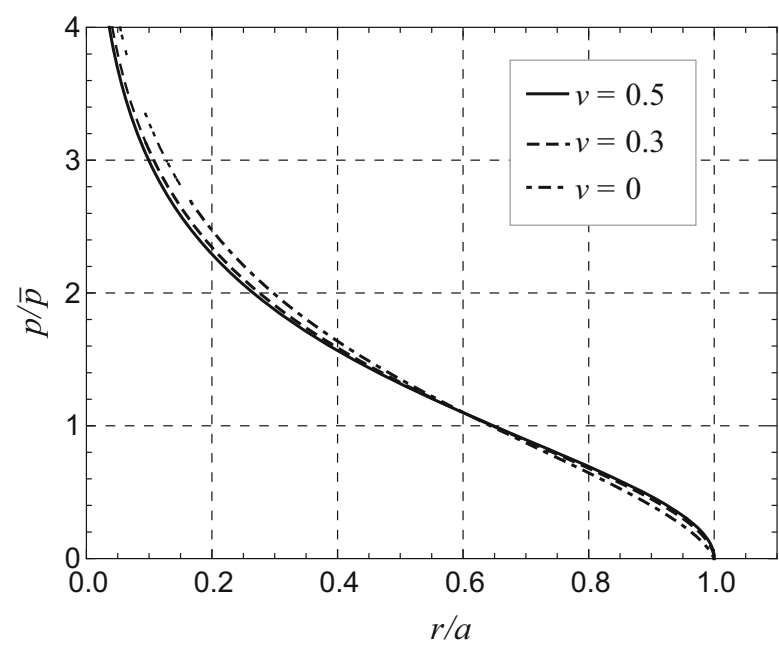

The results for the normal and tangential stresses via numerical integration are visualized in Figs. 2.53 and 2.54. Note again that the differences in the pressure distribution compared to the frictionless contact are minimal. The magnitude of the tangential stresses increases towards the center. As expected, they increase with a decreasing Poisson's ratio.

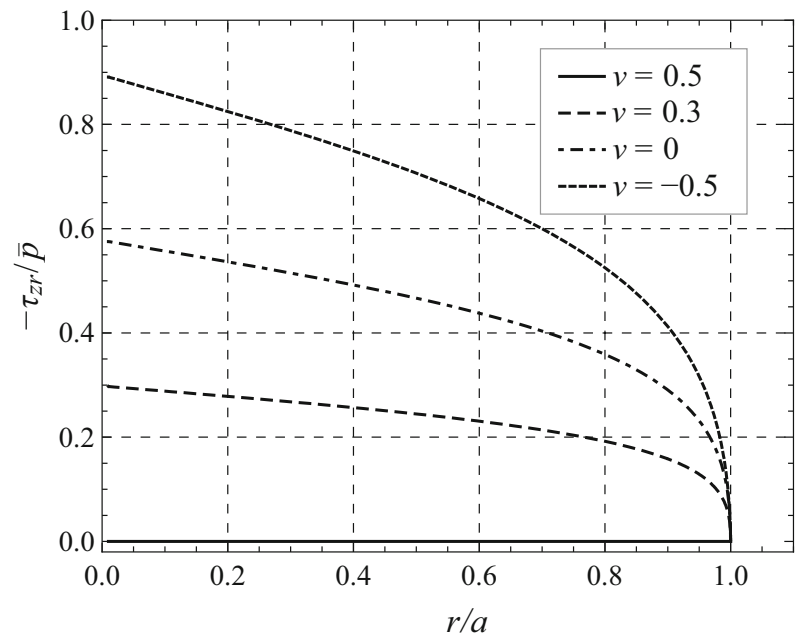

Fig. 2.54 Tangential stresses in the conical contact for different Poisson's ratios $v$, normalized to the average pressure $\bar{p}$ 


\subsubsection{The Paraboloid}

Finally, we will cover the important parabolic contact with complete stick, which can be considered an approximate solution for various curved surfaces, and was solved by Mossakovskii (1963) as well as by Spence (1968). The axially symmetric profile is given by:

$$
f(r)=\frac{r^{2}}{2 R},
$$

where $R$ denotes the curvature radius of the paraboloid. The equivalent onedimensional profile follows from (2.144):

$$
g(x)=\kappa(2, v) \frac{x^{2}}{2 R} .
$$

In contrast to the conical contact, the stretch factor can only be calculated numerically. Selected values can be found in Table 2.2. Spence (1968) did provide a good approximation for the scaling factor:

$$
\begin{aligned}
\kappa(2, v) & \approx \frac{2}{1-0.6931(2 \vartheta)^{2}+0.2254(2 \vartheta)^{4}} \quad \text { with } \\
\vartheta(v) & =\frac{1}{2 \pi} \ln (3-4 v) .
\end{aligned}
$$

The indentation depth can be determined from (2.135), from which we can then derive the condition of the vanishing displacement of the Winkler foundation at the contact edge. Additionally, the normal force can be calculated from the balance of forces in the $z$-direction in accordance with (2.136). The indentation depth and normal force then follows as:

$$
\begin{aligned}
d(a) & =\kappa(2, v) \frac{a^{2}}{2 R} \\
F_{N}(a) & =\frac{4 G}{3 R} \frac{\ln (3-4 v)}{1-2 v} \kappa(2, v) a^{3} .
\end{aligned}
$$

However, analytical solutions of the surface stresses and normal displacements do not appear possible. Although Zhupanska (2009) claimed to have analytically calculated these quantities, her formulas contain series and integral expressions. Taking into account the surface displacement of the Winkler foundation in formulas (2.137), a numerical calculation leads to the solutions for the normal and tangential stresses shown in Figs. 2.55 and 2.56. From the pressure distribution in Fig. 2.55, it is apparent that the pressure maximum in the center of the contact area increases with a decreasing Poisson's ratio. The contact radius decreases simultaneously which, due to the normalization with respect to the contact radius, is not represented in the figure. The tangential stresses are zero in the center and at the edge. As expected, they increase with a decreasing Poisson's ratio. The curve for 


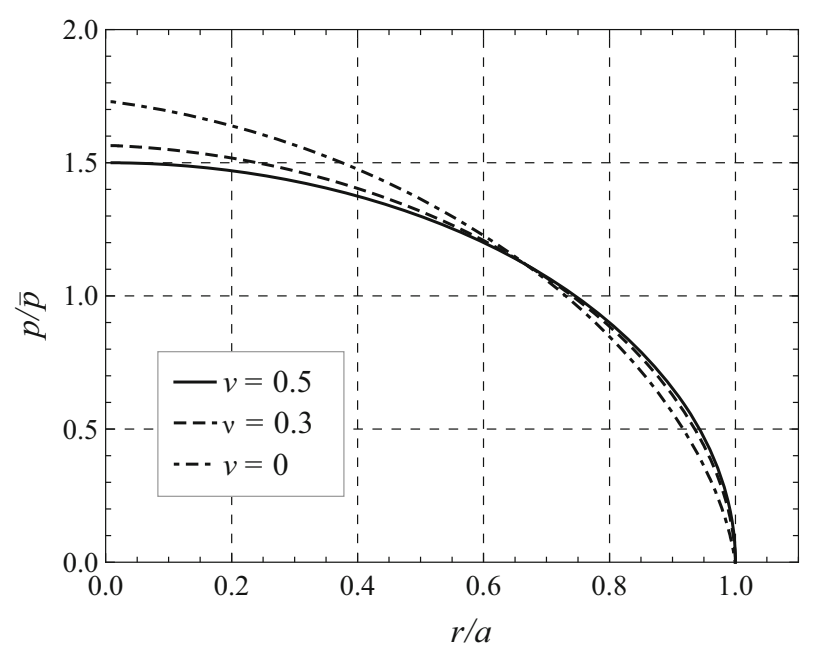

Fig. 2.55 Pressure distribution in the contact with a paraboloid for different Poisson's ratios $v$, normalized to the average pressure

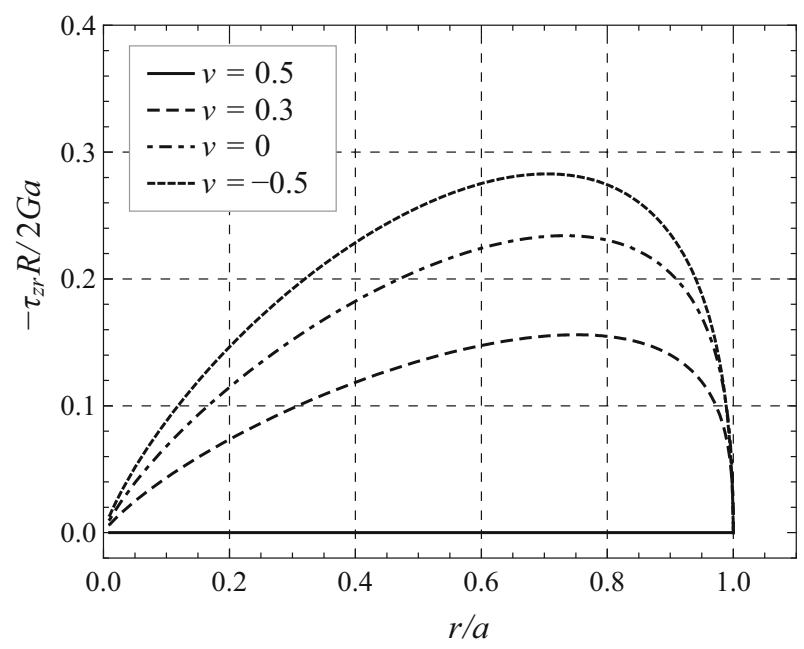

Fig. 2.56 Normalized tangential stresses in the contact with a paraboloid for different Poisson's ratios $v$

$v=0.3$ coincides exactly with Zhupanska's (2009), who utilized torus coordinates for the solution.

For the sake of completeness, the normal surface displacement for several Poisson's ratios is given in a graphical representation in Fig. 2.57. The figure offers a clear illustration of the fact that achieving the same contact area requires a greater indentation depth than for the frictionless contact. 


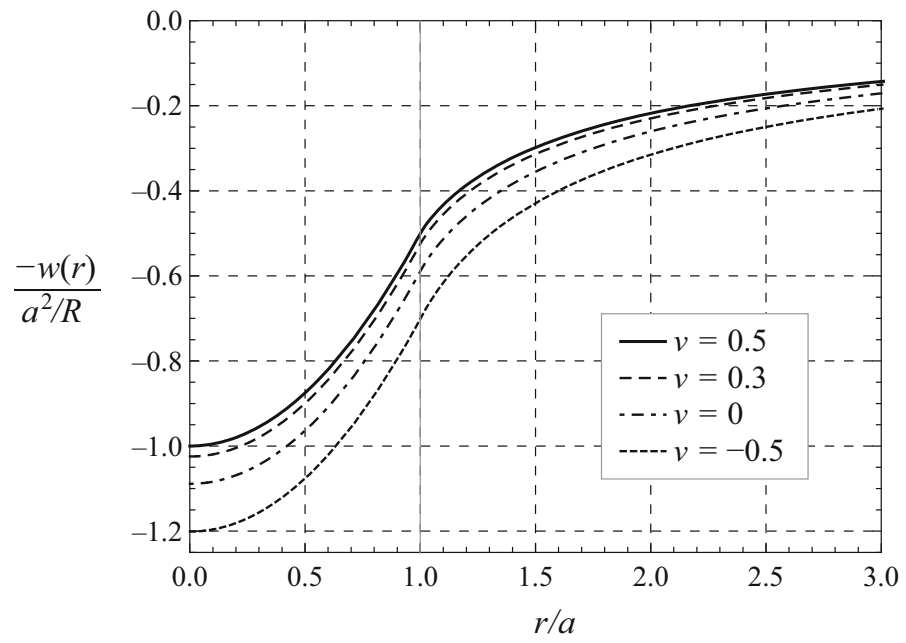

Fig. 2.57 Normalized normal surface displacement of the half-space for indentation by a paraboloid for different Poisson's ratios $v$

\section{References}

Abramian, B.L., Arutiunian, N.K., Babloian, A.A.: On two-contact problems for an elastic sphere. PMM J. Appl. Math. Mech. 28(4), 769-777 (1964)

Argatov, I.I., Guinovart-Díaz, R., Sabina, F.J.: On local indentation and impact compliance of isotropic auxetic materials from the continuum mechanics viewpoint. Int. J. Eng. Sci. 54, 42-57 (2012)

Barber, J.R.: Indentation of the semi-infinite elastic solid by a concave rigid punch. J. Elast. 6(2), 149-159 (1976)

Barber, J.R.: A four-part boundary value problem in elasticity: indentation by a discontinuously concave punch. Appl. Sci. Res. 40(2), 159-167 (1983)

Borodich, F.M., Keer, L.M.: Evaluation of elastic modulus of materials by adhesive (no-slip) nano-indentation. Proc. R. Soc. London Ser. A 460, 507-514 (2004)

Boussinesq, J.: Application des Potentiels a L'etude de L'Equilibre et du Mouvement des Solides Elastiques. Gauthier-Villars, Paris (1885)

Ciavarella, M.: Indentation by nominally flat or conical indenters with rounded corners. Int. J. Solids Struct. 36(27), 4149-4181 (1999)

Ciavarella, M., Hills, D.A., Monno, G.: The influence of rounded edges on indentation by a flat punch. Proc. Inst. Mech. Eng. C J. Mech. Eng. Sci. 212(4), 319-327 (1998)

Collins, W.D.: On the solution of some Axi-symmetric boundary value problems by means of integral equations. VIII. Potential problems for a circular annulus. Proc. Edinb. Math. Soc. Ser. 2 13(3), 235-246 (1963)

Ejike, U.B.C.O.: Contact problem for an elastic half-space and a rigid conical frustum. Proceedings of the First National Colloquium on Mathematics and Physics. (1969)

Ejike, U.B.C.O.: The stress on an elastic half-space due to sectionally smooth-ended punch. J. Elast. 11(4), 395-402 (1981)

Fabrikant, V.I.: Four types of exact solution to the problem of an axi-symmetric punch bonded to a transversely isotropic half-space. Int. J. Eng. Sci. 24(5), 785-801 (1986) 
Föppl, L.: Elastische Beanspruchung des Erdbodens unter Fundamenten. Forsch. Gebiet Ingenieurwes. A 12(1), 31-39 (1941)

Galin, L.A.: Three-dimensional contact problems of the theory of elasticity for punches with a circular planform. Prikladnaya Matem. Mekhanika 10, 425-448 (1946)

Guduru, P.R.: Detachment of a rigid solid from an elastic wavy surface: theory. J. Mech. Phys. Solids 55(3), 445-472 (2007)

Hamilton, G.M., Goodman, L.E.: The stress field created by a circular sliding contact. J. Appl. Mech. 33(2), 371-376 (1966)

Hertz, H.: Über die Berührung fester elastischer Körper. J. Reine Angew. Math. 92, 156-171 (1882)

Huber, M.T.: Zur Theorie der Berührung fester elastischer Körper. Ann. Phys. 14, 153-163 (1904)

Hunter, S.C.: Energy absorbed by elastic waves during impact. J. Mech. Phys. Solids 5(3), 162-171 (1957)

Jordan, E.H., Urban, M.R.: An approximate analytical expression for elastic stresses in flat punch problem. Wear 236, 134-143 (1999)

Lamb, H.: On Boussinesq's problem. Proc. London Math. Soc. 34, 276-284 (1902)

Love, A.E.H.: Boussinesq's problem for a rigid cone. Q. J. Math. 10(1), 161-175 (1939)

Maugis, D., Barquins, M.: Adhesive contact of sectionally smooth-ended punches on elastic halfspaces: theory and experiment. J. Phys. D Appl. Phys. 16(10), 1843-1874 (1983)

Mossakovskii, V.I.: The fundamental mixed problem of the theory of elasticity for a half-space with a circular line separating the boundary conditions. Prikladnaya Matem. Mekhanika 18(2), 187-196 (1954)

Mossakovskii, V.I.: Compression of elastic bodies under conditions of adhesion (axi-symmetric case). PMM J. Appl. Math. Mech. 27(3), 630-643 (1963)

Pharr, G.M., Oliver, W.C., Brotzen, F.R.: On the generality of the relationship among contact stiffness, contact area, and elastic modulus during indentation. J. Mater. Res. 7(3), 613-617 (1992)

Popov, V.L., Heß, M.: Methode der Dimensionsreduktion in Kontaktmechanik und Reibung. Springer, Heidelberg (2013). ISBN 978-3-642-32672-1

Popov, V.L., Heß, M.: Method of dimensionality reduction in contact mechanics and friction. Springer, Heidelberg (2015). ISBN 978-3-642-53875-9

Popov, V.L., Heß, M., Willert, E., Li, Q.: Indentation of concave power-law profiles with arbitrary exponents (2018). https://arXiv.org/abs/1806.05872. cond-mat.soft

Rao, A.K.: Stress concentrations and singularities at interface corners. Z. Angew. Math. Mech. 31, 395-406 (1971)

Schubert, G.: Zur Frage der Druckverteilung unter elastisch gelagerten Tragwerken. Ing. Arch. 13(3), 132-147 (1942)

Segedin, C.M.: The relation between load and penetration for a spherical punch. Mathematika 4(2), 156-161 (1957)

Shtaerman, I.Y.: On the Hertz theory of local deformations resulting from the pressure of elastic bodies. Dokl. Akad. Nauk. SSSR 25, 359-361 (1939)

Shtaerman, I.Y.: Contact problem of the theory of elasticity. Gostekhizdat, Moscow (1949)

Sneddon, I.N.: The relation between load and penetration in the axi-symmetric Boussinesq problem for a punch of arbitrary profile. Int. J. Eng. Sci. 3(1), 47-57 (1965)

Spence, D.A.: Self-similar solutions to adhesive contact problems with incremental loading. Proc. R. Soc. London A Math. Phys. Eng. Sci. 305, 55-80 (1968)

Spence, D.A.: The Hertz contact problem with finite friction. J. Elast 5(3-4), 297-319 (1975)

Zhupanska, O.I.: Axi-symmetric contact with friction of a rigid sphere with an elastic half-space. Proc. R. Soc. London Ser. A 465, 2565-2588 (2009) 
Open Access This chapter is licensed under the terms of the Creative Commons Attribution 4.0 International License (http://creativecommons.org/licenses/by/4.0/), which permits use, sharing, adaptation, distribution and reproduction in any medium or format, as long as you give appropriate credit to the original author(s) and the source, provide a link to the Creative Commons license and indicate if changes were made.

The images or other third party material in this chapter are included in the chapter's Creative Commons license, unless indicated otherwise in a credit line to the material. If material is not included in the chapter's Creative Commons license and your intended use is not permitted by statutory regulation or exceeds the permitted use, you will need to obtain permission directly from the copyright holder. 